\title{
Microstimulation in Visual Area MT: Effects on Direction Discrimination Performance
}

\author{
C. Daniel Salzman, Chieko M. Murasugi, Kenneth H. Britten, and William T. Newsome \\ Department of Neurobiology, Stanford University School of Medicine, Stanford, California 94305
}

Physiological and behavioral evidence suggests that the activity of direction selective neurons in visual cortex underlies the perception of moving visual stimuli. We tested this hypothesis by measuring the effects of cortical microstimulation on perceptual judgements of motion direction. To accomplish this, rhesus monkeys were trained to discriminate the direction of motion in a near-threshold, stochastic motion display. For each experiment, we positioned a microelectrode in the middle of a cluster of neurons that shared a common preferred direction of motion. The psychophysical task was then adjusted so that the visual display was presented directly over the neurons' receptive field. The monkeys were required to discriminate between motion shown either in the direction preferred by the neurons or in the opposite direction. On half the trials of an experiment, we applied electrical microstimulation while monkeys viewed the motion display. We hypothesized that enhancing the neurons' discharge rate would introduce a directionally specific signal into the cortex and thereby influence the monkeys' choices on the discrimination task. We compared the monkeys' performance on "stimulated" and "nonstimulated" trials in 139 experiments; all trials within an experiment were presented in random order. Statistically significant effects of microstimulation were obtained in 89 experiments. In 86 of the 89 experiments with significant effects $(97 \%)$, the monkeys indicated that motion was in the neurons' preferred direction more frequently on stimulated trials than on nonstimulated trials. The data demonstrate a functional link between the activity of direction selective neurons and perceptual judgements of motion direction.

The primate visual system mediates the perception of complex stimuli that vary in shape, color, depth, and movement. One

\footnotetext{
Received Oct. 14, 1991; revised Jan. 10, 1992; accepted Jan. 15, 1992.

We are particularly grateful to Drs. J. A. Movshon and M. N. Shadlen for their contributions to this work. Wc also thank Dr. B. W. Brown for statistical consultations. Drs. B. Wandell, E. A. Deyoe, J. T. McIlwain, and J. H. R. Maunsell made useful suggestions during the course of these experiments, and Dr. S. K. McConnell provided helpful comments on the manuscript. Finally, we are grateful to Judy Stein for valuable technical assistance throughout these experiments. This work was supported by the National Eye Institute (EY-5603), the Office of Naval Research (N00014-88-K-0161), and a McKnight Development Award to W.T.N. C.D.S. was supported by a Medical Student Research Fellowship from the Howard Hughes Medical Institute. K.H.B. receives support from NIH Training Grant NS 07158-11, and C.M.M. is a postdoctoral fellow supported by the Natural Sciences and Engineering Research Council of Canada.

Correspondence should be addressed to William T. Newsome, Associate Professor, Department of Neurobiology, Fairchild Building, D209, Stanford University School of Medicine, Stanford, CA 94305.

Copyright (C) 1992 Society for Neuroscience $0270-6474 / 92 / 122331-25 \$ 05.00 / 0$
}

step toward understanding this process is to characterize how neurons respond to different visual stimuli. Accordingly, singleunit recordings have demonstrated that cortical neurons are specialized to encode information about distinct aspects of a visual stimulus. In striate cortex, for example, neurons may respond selectively to the orientation of local contours, to the color of a visual stimulus, to contours falling on disparate locations of the two retinas, or to motion of a particular direction (e.g., Hubel and Wiesel, 1962, 1968; Barlow, 1967; Schiller, 1976; Fischer and Poggio, 1979; De Valois, 1982; Livingstone and Hubel, 1984; Lennie et al., 1990). A common assumption is that the physiological properties of neurons reflect their role in mediating visual perception. Such "linking hypotheses" connect the perception of form, color, depth, and motion to neurons that respond selectively to particular orientations, wavelengths, disparities, and directions of motion (c.g., Tcller, 1984). Despitc their substantial impact on contemporary vision research, evidence confirming these hypotheses is actually quite sparse.

Current efforts to link single neuron properties to specific perceptual capabilities are facilitated by the fact that neurons are organized into larger anatomical units that are physiologically specialized. In the superficial layers of striate cortex, for example, orientation selective neurons predominate in the cytochrome oxidase-free regions while nonoriented cells are more common in cytochrome oxidase-dense regions (Livingstone and Hubel, 1984). Furthermore, columns of orientation selective neurons are selectively interconnected with neighboring columns that have similar physiological properties, thus creating local circuits devoted to the analysis of particular orientations (Ts'o et al., 1986; Ts'o and Gilbert, 1988; Gilbert and Wiesel, 1989). Physiological specialization occurs at the level of cortical areas as well. Primate visual cortex is composed of morc than 20 different cortical areas, and several of these areas appear to have distinctive properties (e.g., Zeki, 1978; Ungerleider and Mishkin, 1982; Mishkin et al., 1983; Maunsell and Newsome, 1987). Thus, physiological specialization at several levels-single neurons, local circuits, and visual areas-suggests that different cortical pathways mediate particular aspects of visual perception. This notion is consistent with clinical observations of highly specific deficits following local cortical damage (e.g., Ratcliff and Davies-Jones, 1972; Meadows, 1974a,b; Damasio and Benton, 1979; Pearlman et al., 1979; Damasio et al., 1982; Zihi et al., 1983; Joynt et al., 1985; Plant, 1990).

The most compelling evidence for functional specialization in primate visual cortex is found in a system of closely connected visual areas that is predominantly concerned with the analysis of visual motion information. These areas comprise a cortical 
pathway extending from layer $4 \mathrm{~B}$ of striate cortex, through dorsal extrastriate cortex, and on to the parietal lobe (for review, see Maunsell and Newsome, 1987). Direction selectivity is the salient physiological feature of neurons within this pathway, and the initial inference of a functional specialization for motion vision rested solely on the hypothesis linking direction selectivity to motion perception (Dubner and Zeki, 1971; Zeki, 1974).

The middle temporal area (MT, or V5), an extrastriate area within the motion pathway, provides a particularly convenient target for experimental investigation. Anatomically, MT lies in the posterior bank of the superior temporal sulcus (STS) and has a columnar organization analogous to other cortical areas. Physiological recording has demonstrated that over $80 \%$ of MT neurons are directionally selective (Zeki, 1974; Baker et al., 1981; Van Essen et al., 1981; Maunsell and Van Essen, 1983; Albright, 1984) and that neurons within a single column in MT have similar receptive field locations and preferred directions (Albright et al., 1984). The preferred direction of motion and receptive field location vary systematically from column to column so that all directions of motion at each point in the contralateral visual field are represented. In addition, the direct connections between striate cortex and MT make robust visual responses relatively easy to obtain.

To examine the relationship between the responses of MT neurons and motion perception, we conducted lesion and single neuron recording experiments in monkeys trained to discriminate the direction of motion in a near-threshold, stochastic motion display. Using this general approach, we and others demonstrated that lesions of MT selectively elevate perceptual thresholds on motion discrimination or detection tasks (Newsome and Pare, 1986, 1988; Siegel and Andersen, 1986). Further studies showed that many MT neurons were sufficiently sensitive to motion signals in the stochastic display to account for the monkeys' psychophysical performance measured on the same set of trials (Newsome et al., 1989a). Moreover, the response amplitude of some MT neurons was correlated with the monkey's choices on a trial-by-trial basis (Newsome et al., 1989b). These observations strongly suggested that the directional signals carried by MT neurons underlie judgements of motion direction.

To establish this relationship more firmly, we have now attempted to influence a monkey's choices on the direction discrimination task by modifying the activity of MT neurons with electrical microstimulation. The methodological challenge of these experiments was to activate selectively neurons that encoded a similar direction of motion. To accomplish this, we positioned the stimulating electrode within a cluster of neurons having a common preferred direction. We then applied smallamplitude stimulating pulses while the monkey performed a direction discrimination task. The predominant effect of microstimulation was to bias the monkey's decisions in favor of the direction preferred by neurons at the stimulation site. The data therefore establish a causal relationship between the activity of the stimulated neurons and perceptual judgements of motion direction. This result provides direct support for the linking hypothesis associating direction selectivity with motion perception and demonstrates a major functional role of the "motion pathway" within visual cortex.

Brief reports of these experiments have appeared previously (Newsome et al., 1990, 1991; Salzman et al., 1990a,b; Salzman and Newsome, 1991).

\section{Materials and Methods}

We conducted microstimulation experiments in four hemispheres of three rhesus monkeys (Macacca mulatta) weighing between 6 and 11 $\mathrm{kg}$. Prior to the experiments, each monkey was surgically implanted with a head-holding device, an eye coil, and a recording cylinder placed over a craniotomy above the occipital lobe (Evarts, 1966, 1968; Judge et al., 1980). All surgeries were performed under aseptic conditions with halothane anesthesia. In daily experimental sessions, a monkey was seated comfortably in a primate chair with its head fixed in place. The chair was positioned within a magnetic search coil apparatus that allowed precise monitoring of the monkey's eye movements (Robinson, 1963; Fuchs and Robinson, 1966). At the end of an experimental session, the monkey was returned to its home cage.

Visual stimuli. We required the monkeys to discriminate the direction of motion in a dynamic random dot display shown on a CRT screen placed $57 \mathrm{~cm}$ in front of the monkey. A PDP $11 / 73$ computer controlled the display. The essential feature of the stimulus is that the experimenter can control the strength of the motion signal in the display. Each dot in the stimulus is plotted on the CRT screen and lives for approximately $20-30 \mu \mathrm{sec}$. Only one dot is presented per $150 \mu \mathrm{sec}$, but persistence within the visual system is such that many dots appear to be present simultaneously. The probability that a given dot will be replotted at a specified location in space and in time relative to its previous location determines the strength of the motion signal. At one extreme, shown on the right side of Figure 1, the probability that a dot will be replotted at a constant offset in space and time is 1.0 ; this is the strongest motion signal provided by this stimulus, and we refer to it as the $100 \%$ correlation state. At the other extreme, the probability that a dot will be replotted at a constant offset in space and time is 0 . There is no net motion signal contained in this stimulus, and it is referred to as $0 \%$ correlation. The visual impression created by a $0 \%$ correlation stimulus resembles the noise observed on a television set tuned between channels, with many dots flickering on and off at random locations on the screen. Typically, the monkeys discriminated the direction of motion for correlation levels between these two extremes. The middle panel of Figure 1 , for example, depicts the $50 \%$ correlation level. Half of the dots provide the correlated motion signal, while the other half comprise motion noise.

This stochastic visual display offers several advantages for studying the neural mechanisms devoted to motion analysis. First, since there are no repeated edges or boundaries within the stimulus, successful discrimination cannot be performed by inferring the direction of motion from positional cues (Braddick, 1974; Morgan and Ward, 1980; Nakayama, 1981; Williams and Sekuler, 1984). To discriminate accurately the direction of motion in the stimulus, the observer must rely completely on primary motion sensing mechanisms within the brain. Second, the experimenter can vary a number of the parameters of the stimulus, allowing for physiological analysis of direction selective neurons as well as psychophysical measurements of direction discrimination thresholds. By changing the direction and amplitude of the spatial offset between correlated dots, for example, the optimal velocity for the neurons under study can be determined. For all experiments in the present study, the temporal offset between correlated dots was held constant at $45 \mathrm{msec}$. This approximately matches the optimum temporal offset for human short-range motion perception (Braddick, 1974; Morgan and Ward, 1980) and is effective for eliciting direction-selective responses from MT neurons (Mikami et al., 1986). Finally, the variable-strength motion display permitted us to conduct experiments near psychophysical threshold. With the monkey working near the limits of performance, small changes in the discharge rate of neurons contributing to the perceptual process are more likely to affect decisions in the direction discrimination task.

Recording and microstimulation. At the beginning of each recording session, we mounted an hydraulic microdrive on the recording cylinder. This microdrive controlled electrode advance into the cortex. We located MT using either glass-coated platinum-iridium microelectrodes (impedances, $0.5-1.0 \mathrm{M} \Omega$ at $1 \mathrm{kHz}$ ) or tungsten microelectrodes (Micro Probe, Inc.) coated with parylene and having an exposed tip length of 20-30 $\mu \mathrm{m}$ (impedances, $0.5-1.5 \mathrm{M} \Omega$ ). The platinum-iridium electrodes could puncture dura; the tungsten electrodes were inserted into a guide tube that had been pushed through dura. A plastic grid with holes spaced $1 \mathrm{~mm}$ apart supported the guide tube (Crist et al., 1988). Electrical signals from the electrode were amplified and displayed on an oscilloscope and an audio monitor. 

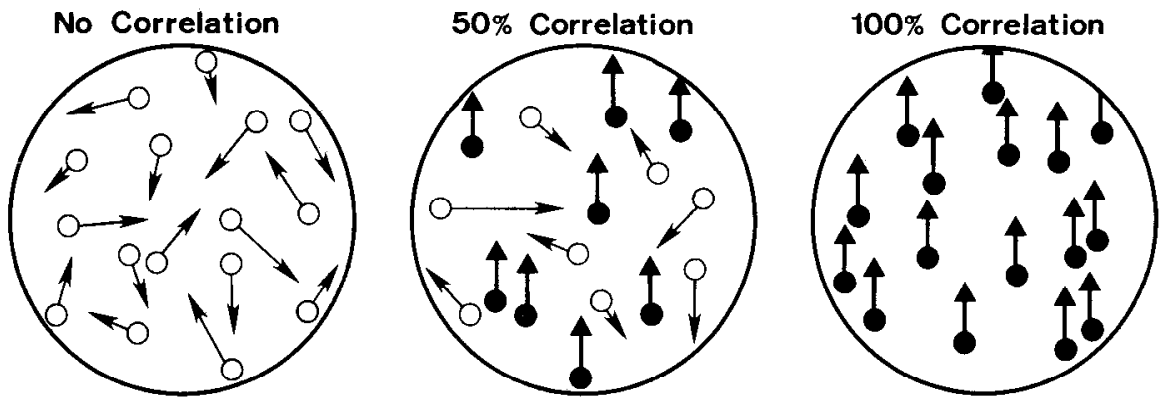

Figure 1. Diagram of the motion stimulus used in the present experiments. The stimulus is composed of a sequence of dots plotted on a CRT screen. The strength of the motion stimulus is determined by the probability that a given dot will be replotted at a fixed spatial and temporal interval. Right panel, The strongest motion signal achieved by the stimulus. The probability that a dot will be replotted at a constant spatial and temporal offset is 1 . We refer to this condition as the $100 \%$ correlation level. Left panel. The $0 \%$ correlation level. Each dot is replotted at a random location on the CRT screen. Middle panel, The $50 \%$ correlation level. The probability that a dot is replotted at a fixed spatial and temporal interval is 0.5. Thus, half the dots appear to carry a unidirectional motion signal, and the remaining dots create a masking motion noise.

Our angle of approach to MT was approximately $20^{\circ}$ above the horizontal plane, commencing in striate cortex, passing across the lunate sulcus, and then onto the posterior bank of the superior temporal sulcus (STS). We identified MT by the characteristic dircction sclectivity of its neurons and the size and eccentricity of its receptive fields.

Microstimulation was applied through the tungsten microelectrodes described above. To decrease electrode impedance and facilitate passage of current, we gold-plated the tip of the microelectrode before inserting it into the guide tube. The impedance of the gold-plated electrodes measured in the brain was usually $0.3-0.7 \mathrm{M} \Omega$. These low-impedance electrodes were well suited for recording from multineuron clusters, a process that was important for selection of stimulation sites (see below).

We stimulated selected sites in MT using a pulse generator in series with an optical stimulus isolation unit. The stimulating pulses were biphasic, cathodal pulse leading. Each pulse was $0.2 \mathrm{msec}$ in duration with a $0.1-\mathrm{msec}$ between the pulses. For the first 11 experiments, the stimulation pulses were $10 \mu \mathrm{A}$ in amplitude and $500 \mathrm{~Hz}$ in frequency. For the remaining experiments described in this article, we reduced the pulse pair frequency to $200 \mathrm{~Hz}$. This frequency matches the largest responses that we have observed in MT neurons (Britten, Movshon, Shadlen, and Newsome, unpublished observations).

Selection of stimulation sites. Our goal in these experiments was to activate selectively neurons that had a similar preferred direction. We therefore attempted to place the electrode in the middle of a cluster of neurons having similar physiological properties. Microstimulation parameters were selected to restrict current spread to neurons near the stimulation site. We know of no quantitative studies of the spread of microstimulation current in primate visual cortex. In motor cortex, however, Stoney et al. (1968) have estimated that a single $10 \mu \mathrm{A}$ cathodal pulse activates neurons within approximately $85 \mu \mathrm{m}$ of the electrode tip $(95 \%$ confidence limits at $70 \mu \mathrm{m}$ and $120 \mu \mathrm{m})$. From these data, we infer that the $10 \mu \mathrm{A}$ pulses employed in the present study directly activated neurons within a sphere roughly $140-240 \mu \mathrm{m}$ in diameter. We therefore selected stimulation sites in which neurons encountered over at least $200 \mu \mathrm{m}$ of electrode travel had similar preferred directions, preferred speeds, and receptive field locations. We also required that the neurons yield vigorous, directional responses to the random dot stimulus described above. To maximize our chances of achieving selective activation, we measured the length of the stimulation site and positioned the electrode at roughly the midpoint of the site. Nonetheless, uncertainties regarding the extent of current spread and the local geometry of direction columns near the electrode tip hampered our ability to restrict activation to the targeted neurons. These issues will be considered in detail in the Discussion.

Behavioral paradigm. By elevating the discharge rate of neurons encoding a particular direction of motion, we attempted to influence the monkey's choices on the two-alternative, forced-choice direction discrimination task depicted in Figure 2. We measured the effects of microstimulation by comparing a monkey's choice performance on stimulated and nonstimulated trials. To maximize the likelihood that the monkey would base its judgements of motion direction on the signals carried by neurons at the stimulation site, we tailored the task so that the demands of the psychophysical task matched the information supplied by neurons at the stimulation site. Thus, upon selecting a stimulation site, we mapped the multiunit receptive field (Fig. $2 A$, inner, shaded circle) and determined the preferred direction (arrow) and preferred speed of the neurons. A visual stimulus aperture approximately equal in size to the neurons' receptive field was placed over the CRT screen (outer circle). We then positioned the fixation point (Fig. $2 A$, FP) so that the receptive field was superimposed on the visual stimulus aperture when the monkey directed its gaze toward the fixation point. Motion signals were presented within the visual stimulus aperture over a range of correlation levels near psychophysical threshold. Motion occurred in either the preferred direction or the null (opposite) direction of the neurons at the stimulation site, and the speed of the motion signals was closely matched to the optimal speed of the neurons.

To perform the motion discrimination task, the monkey centered its gaze at the fixation point. After the monkey fixated the target, visual stimulus presentation began and lasted for $1 \mathrm{sec}$. After $1 \mathrm{sec}$, the fixation point and visual display were extinguished, and two light-emitting diodes appeared corresponding to the two possible directions of motion (Fig. 2A, Pref LED and Null LED). The monkey reported its judgement of motion direction by making a saccadic eye movement to one of the two LEDs. We rewarded the monkey for correct choices with a drop of water or juice. If the monkey broke fixation prematurely, or if it failed to indicate a choice by making an appropriate saccade, the trial was aborted and the data discarded. We defined broken fixation to be any departure of eye position from the center of the fixation point that exceeded $1.5^{\circ}$.

Figure $2 B$ depicts the timing of events on a microstimulation trial. As in the nonstimulated trial described above, the monkey viewed the motion display for $1 \mathrm{sec}$ after centering its gaze at the fixation point. The train of electrical stimulating pulses began simultaneously with the onset of the visual stimulus. Microstimulation ended when the visual stimulus and fixation point were extinguished, and the monkey reported its judgement of motion direction in the same manner as described for nonstimulated trials. We continued to reward the monkey only for correct choices on stimulated trials. Thus, the incentive for the monkey on both stimulated trials and nonstimulated trials was to discriminate correctly the direction of motion in the visual stimulus; there was no incentive to make an excess of decisions in favor of either direction.

We used the method of constant stimuli to measure psychophysical thresholds. Each experiment contained 640 trials, half with electrical microstimulation and half without. For both subsets, motion occurred in the neurons' preferred direction on half of the trials and in the null direction on the remaining half. We usually collected data at four correlation levels: three near psychophysical threshold, and one at $0 \%$ correlation. At $0 \%$ correlation, where there was no net motion signal in the display, the monkey was rewarded with a probability of 0.5 for either choice. The highest correlation level was chosen so that the monkey would make $80-90 \%$ correct choices; the monkey therefore received rewards on approximately $60-70 \%$ of the trials overall in an experiment. Since the monkey could achieve a $50 \%$ reward rate simply by guessing, the $60-70 \%$ rate was necessary to ensure that the monkey would work 


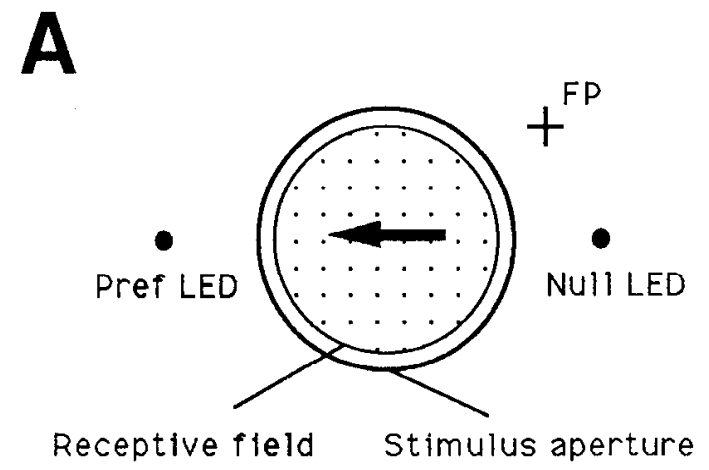

B

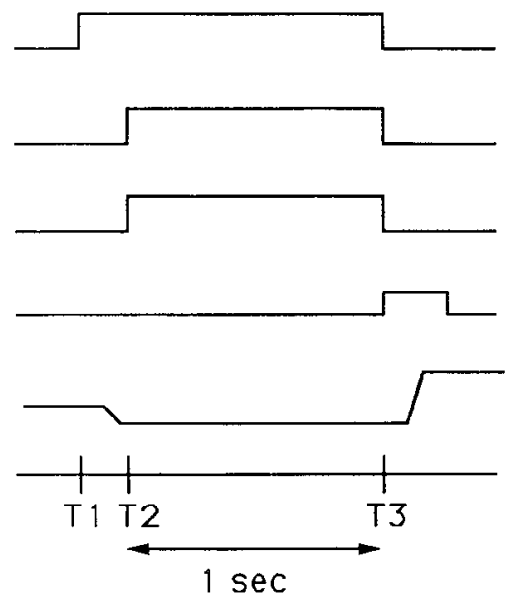

Fixation Point

Vis. Stimulus

Elect. Stimulus

Target LEDs

Eye Position

Time

Figure 2. The behavioral paradigm used to measure the effects of microstimulation. $A$, A diagram showing the receptive field of neurons at the stimulation site (inner, stippled circle), the preferred direction of the neurons (arrow), the visual stimulus aperture within which the visual stimulus was presented (outer circle), the fixation point $(F P)$, and the two LEDs (Pref LED and Null LED). Experiments were conducted over a broad range of eccentricities, receptive field sizes, and preferred directions. $B$, Temporal sequence of events in a trial containing microstimulation. To perform the task in the stimulated condition, the monkey fixated, and the visual stimulus then appeared for $1 \mathrm{sec}$ commencing at time $T 2$. The train of electrical pulses began simultaneously with the onset of the visual stimulus. At time $T 3$, the fixation point, visual stimulus, and microstimulation were extinguished, and the monkey indicated its choice by making a saccade to one of the two LEDs.

hard at the discrimination task throughout the experiment. All trial conditions were presented in random order.

Throughout the training of the monkeys and throughout these experiments, a correction procedure was used to discourage spatial choice biases toward one or the other LED. A monkey entered a "correction loop" upon making three consecutive incorrect choices in the same direction. Motion was presented consistently in the neglected direction until the monkey correctly chose that direction. No microstimulation was applied on correction loop trials, and data from these trials were not included in the analysis. In practice, the monkeys entered correction loops frequently during the early stages of training, but rarely in the late stages of training.

For all experiments, behavioral control and data acquisition were accomplished using a real-time experimental system developed by Hays et al. (1982) at the Laboratory of Sensorimotor Research of the National Eye Institute.

Stimulation site data base. Unless otherwise noted, the results pre- sented in this article are based upon an original data base of 62 experiments employing the behavioral paradigm described above. The data were obtained from three monkeys: 38 experiments in monkey $\mathrm{J}, 8$ in monkey $\mathrm{L}$, and 16 in monkey $\mathrm{E}$. In Figure $6 B$ we have included additional data taken from a later series of 77 experiments that will be referred to as the "expanded data set." Each of these 77 experiments included a subset of trials corresponding to the basic paradigm outlined above. We include these data in Figure $6 B$ simply to corroborate the primary finding of this article with a substantially larger number of experiments.

Among the 77 experiments included in the expanded data set, we examined the topographic specificity of the microstimulation effect in 31 experiments by applying microstimulation to neurons in MT whose receptive fields did not correspond to the location of the visual stimulus presentation (section II of Results). In addition, 46 experiments were conducted over an extended range of correlation levels to examine the effect of microstimulation on the slope of psychometric function (section I of Results). In 40 microstimulation experiments not included in the expanded data set, we applied microstimulation prior to the presentation of the visual stimulus (section III of Results). These data are excluded from the expanded data set since the experiments did not include trials in which microstimulation was applied simultaneously with the visual stimulus.

Data analysis. The specific hypothesis underlying the microstimulation experiments was that selective activation of neurons encoding a similar direction of motion should bias a monkey's choices toward the neurons' preferred direction. In other words, microstimulation should increase the number of "preferred decisions" made by the monkey, a preferred decision being defined as a decision in favor of the preferred direction of neurons at the stimulation site. Since the data of primary interest are changes in the proportion of preferred decisions, many data figures will incorporate plots of the proportion preferred decisions as a function of the strength of the motion signal expressed in percentage of correlated dots. An example of this type of plot is illustrated in Figure 3 . The circles show the monkey's performance on the direction discrimination task in the absence of microstimulation. The abscissa in this graph is labeled with positive and negative values to indicate the direction and strength of the motion stimulus. Positive correlation values correspond to motion in the preferred direction, and negative correlation values indicatc motion in the null direction. At high correlation values with motion in the preferred direction, the monkey made preferred decisions on all trials and therefore performed at $100 \%$ correct. At high correlation values but with motion in the null direction, the monkey made no preferred decisions and therefore performed at $100 \%$ correct as well. As the correlation level decreased from either extreme, the monkey's performance deteriorated so that it performed approximately at chance levels ( $50 \%$ correct) at $0 \%$ correlation. Since there is no net motion signal at $0 \%$ correlation, we would expect a monkey to make on average $50 \%$ preferred decisions at this level unless the monkey had a "choice bias" toward one direction or the other. In the experiment depicted, the monkey actually exhibited a choice bias in favor of the preferred direction, and it made approximately $60 \%$ preferred decisions at $0 \%$ correlation.

One might anticipate at lcast two possible influences of microstimulation on the monkeys' choice behavior in our psychophysical paradigm. As noted above, microstimulation might enhance the intracortical signal corresponding to the preferred direction of neurons at the stimulation site, thereby biasing the monkeys' choices toward the preferred direction of the stimulated neurons. In this case, the proportion of preferred decisions would increase at intermediate correlation levels, resulting in a leftward shift of the psychometric function, as illustrated by curve $\mathrm{A}$ in Figure 3. Alternatively, microstimulation might disrupt normal neural processing of motion signals by indiscriminately activating neurons in several columns encoding different directions of motion. In this case, microstimulation would add noise to the underlying neural processes, thereby decreasing the monkey's sensitivity to the visual stimulus. The net result would be to elevate psychophysical threshold and flatten a portion of the psychometric function symmetrically about the $0 \%$ correlation point (see curve B in Fig. 3). Of course microstimulation might add both signal and noise, in which case the "stimulated" psychometric function would be shifted to the left and flattened as compared to the "nonstimulated" psychometric function.

To analyze these data, we employed logistic regression analysis (Cox, 1970), which models the log-odds-ratio of making a preferred decision as a linear sum of the contributions from several sources. The log-odds- 
ratio is simply the logarithm of the ratio of preferred decisions to null (or opposite) decisions. This model was particularly convenient for our purposes since it permitted us to examine the dependence of this odds ratio on the strength of the motion signal, microstimulation, and their interactions. Equation 1 describes this relationship:

$$
\ln \left\langle\frac{P}{1-P}\right\rangle=\beta_{0}+\left(\beta_{1} \cdot \operatorname{stim}\right)+\left(\beta_{2} \cdot \operatorname{corr}\right)+\left(\beta_{3} \cdot \operatorname{stim} \cdot \text { corr }\right),
$$

or equivalently,

$$
P=\frac{1}{1+\exp \left[-\left(\beta_{v}+\left(\beta_{1} \cdot \operatorname{stim}\right)+\left(\beta_{2} \cdot \operatorname{corr}\right)+\left(\beta_{3} \cdot \operatorname{stim} \cdot \operatorname{corr}\right)\right)\right]},
$$

where $P$ corresponds to the probability of making a preferred decision and $\beta_{0}, \beta_{1}, \beta_{2}$, and $\beta_{3}$ are the coefficients to be fit by the regression. Equation 2 has the form of a sigmoidal function and is employed in Figure 3. $\beta_{0}$ is a coefficient that represents the relative contribution of choice bias to the log-odds of making a preferred decision. When the monkey has no choice bias, the value of this coefficient is $0 . \beta_{1}$ is a coefficient representing the effect of microstimulation on the monkey's choice bias. If microstimulation adds signal by activating neurons with similar preferred directions, its effects would be modeled by this term. $\beta_{1}$ is multiplied by "stim," which is equal to 0 on nonstimulated trials and 1 on stimulated trials. $\beta_{2}$ models the effect of stimulus correlation on the monkey's choice behavior and graphically represents the slope of the psychometric function. "Corr" is the correlation level of the motion stimulus. We represent motion in the null direction as negative correlations, since null-direction stimuli will decrease the proportion preferred decisions made. We observed that the log-odds-ratio of a preferred decision usually varied linearly with correlation, thus lending justification to the use of the logistic regression model to describe our data: $\beta_{3}$ is the coefficient corresponding to the possible interaction between microstimulation and the strength and direction of the motion signal. If microstimulation adds noise to the neural processing underlying the monkey's performance on this task, then this effect will be reflected in this term. Since the variable "stim" in Equations 1 and 2 is equal to either 0 or 1 , it is convenient to plot $P$ as a function of correlation separately for stimulated and nonstimulated trials. This convention is observed throughout the article (e.g., Fig. 4).

Logistic regression uses maximum likelihood fitting methodology (Cox, 1970) to evaluate the statistical significance of all the factors contributing to the monkey's decisions. Furthermore, it provides an estimate of the relative contribution of each cocfficient, thus allowing the effect of microstimulation to be expressed in terms equivalent to the effect of stimulus correlation. If a coefficient from the fit function differs significantly from 0 , we may conclude that the factor modeled by that coefficient contributes significantly to the probability of making a preferred decision. For over $80 \%$ of our experiments, $\beta_{3}$ was not significantly different from 0 , indicating that the effect of microstimulation on the log-oddsratio was independent of the strength and direction of the motion signal. Graphically, this means that a pair of sigmoid functions identical in shape may be used to represent the stimulated and nonstimulated conditions. These functions can differ, however, in their placement along the abscissa. Since microstimulation infrequently changed the shape of the psychometric function, we refit the data with a logistic regression model that did not include the interaction term. The equation for this reduced model was

$$
P-\frac{1}{1+\exp \left[-\left(\beta_{0}+\left(\beta_{1} \cdot \operatorname{stim}\right)+\left(\beta_{2} \cdot \operatorname{corr}\right)\right)\right]}
$$

All of the coefficients in this model have the same meaning as in the previous model. The only difference between the models is that the shapes of the stimulated and nonstimulated psychometric functions are now constrained to be identical. For the majority of experiments $(>80 \%)$ reducing the number of free parameters in the model in this manner did not cause the fit of the logistic regression model to be rejected.

Histology. Two of the monkeys from this study were killed with a lethal dose of pentobarbital and perfused with normal saline followed by $10 \%$ formalin (the other monkey is alive and is being employed in related physiological recording experiments). After equilibrating the brains in a $30 \%$ sucrose solution, we sectioned the brains in the sagittal plane. One series of sections was stained for cell bodies with cresyl violet, and a second series was stained for myelinated fibers by the

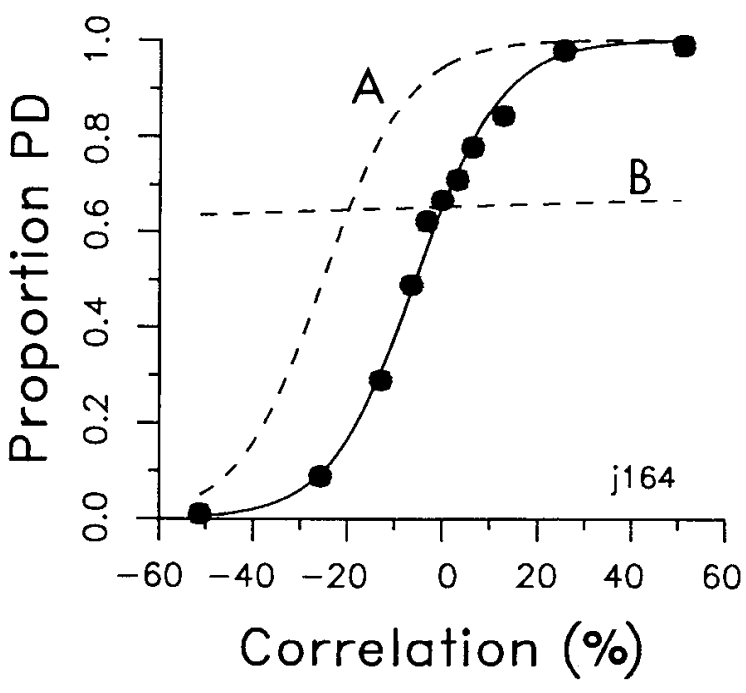

Figure 3. A sample psychometric function in which the proportion of decisions favoring the neurons' preferred direction (preferred decisions, $P D$ ) is plotted as a function of the strength of the motion signal expressed in percentage of correlated dots. The abscissa has been "opened" to indicate separately motion in the preferred direction (positive values) and motion in the opposite or "null" direction (negative values). The solid circles arc rcal data taken from a physiological recording experiment in which no microstimulation was applied. The solid, sigmoidal curve represents the logistic regression function describing the monkey's psychophysical performance. The y-intercept of this function reflects the predicted performance at $0 \%$ correlation, which is indicative of a monkey's choice bias within an experiment. The two broken curves marked $A$ and $B$ represent two possible outcomes of applying microstimulation. If microstimulation adds signal to the neural processes that underlie perceptual judgements of motion direction, we would expect to bias the monkey's choices toward the neurons' preferred direction and thereby shift the psychometric function to the left, as for curve $A$. This function has the same shape as the solid curve. Alternatively, if microstimulation injects noise into the same neural processes, we would expect to flatten the psychometric function, as for curve $B$. In this case, the shape of the function has been changed, but the choice bias ( $y$ intercept) has remained constant. A combination of adding signal and noise might also occur.

method of Gallyas (1979). Reconstruction of guide tube scars and electrode tracks confirmed that microstimulation experiments occurred within visual area MT in both monkeys. In monkey L, only eight microstimulation experiments were performed over a duration of 2 weeks because this monkey became acutely ill unexpectedly. While the animal's death was unfortunate, it provided an opportunity to examine the cortex for possible electrolytic cell damage from the recently conducted microstimulation experiments. We examined every histological section through MT in monkey $\mathrm{L}$ to determine whether our microstimulation paradigm caused inadvertent damage to nearby cells, but we were able to locate only one small lesion (about $30 \mu \mathrm{m}$ diameter) that appeared electrolytic in nature. We therefore believe that the application of microstimulation in these experiments did not result in consistent or substantial damage to nearby neurons. In monkey $J$, we deliberately placed 11 electrolytic lesions in MT during the final 4 weeks of the monkey's life. The lesions were made by applying $20 \mu \mathrm{A}$ of direct current for 10 or $20 \mathrm{sec}$. These lesions marked experiments in which microstimulation had a large effect on the monkey's psychophysical performance. For each lesion in MT, we made a companion lesion on the anterior bank of the superior temporal sulcus to facilitate reconstruction of the electrode tracks.

\section{Results}

Results will be presented in three sections. We first describe the basic effects of microstimulation on psychophysical perfor- 

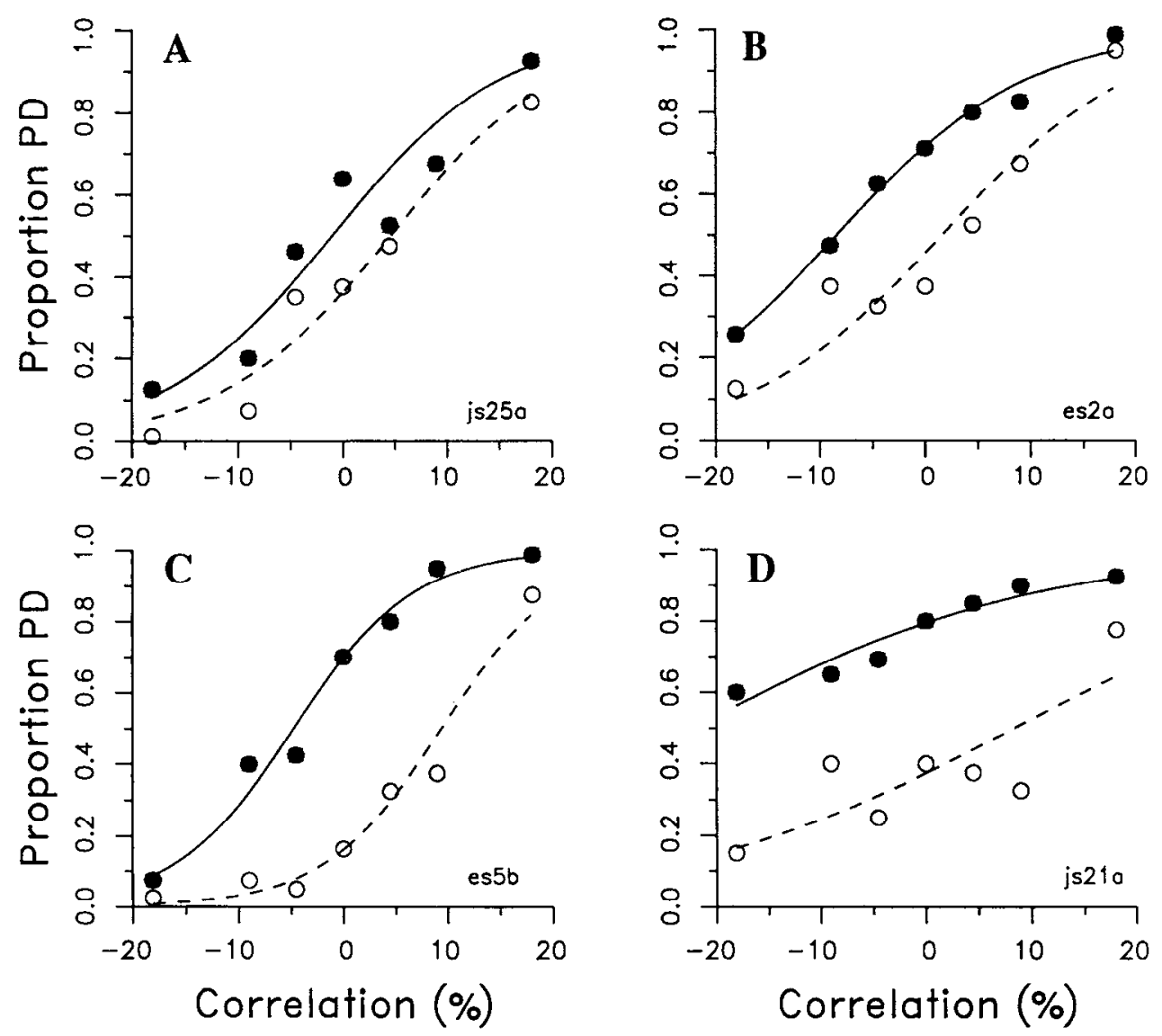

Figure 4. Effect of microstimulation on psychophysical performance for four stimulation sites in MT. The ordinate and abscissa are the same as in Figure 3. Trials that did not contain microstimulation are shown with open symbols; trials that contained microstimulation are represented with solid symbols. This convention for labeling stimulated and nonstimulated data is maintained throughout the article (e.g., Figs. 8, 11, 12, 13, 15, and 16). Each data point is based on 40 trials, except for $0 \%$ correlation, where 80 trials were conducted. The psychometric functions depicted were fitted with Equation 3 (see Materials and Methods) in which the shapes of the stimulated and nonstimulated psychometric functions were constrained to be identical. $A$, A small but significant ( $p \leq 0.0001$, logistic regression analysis) effect of microstimulation in monkey $\mathrm{J}$ (equivalent visual stimulus $=5.6 \%$ correlated dots). $B, \mathrm{~A}$ medium-sized effect in monkey $\mathrm{E}$ (equivalent visual stimulus $=10.0 \%$ correlated dots). $C$, A larger effect of microstimulation in monkey $\mathrm{E}$ (equivalent visual stimulus $=14.2 \%$ correlated dots). $D$, A large effect of microstimulation in monkey $\mathrm{J}$ (equivalent visual stimulus $=30.6 \%$ correlated dots). The difficulty of each experiment varied depending upon several variables, including the eccentricity of the motion display, the size of the visual stimulus aperture, and the speed of the motion stimulus. The variability in difficulty is reflected by the different slopes in each experiment. More difficult discriminations imply that the monkey made fewer correct choices, and the slope of the psychometric function therefore appears flatter (e.g., $D$ ).

mance. Next, we present data that address the issue of mechanisms underlying the microstimulation effects. Finally, we consider an interesting behavioral effect observed on trials that did not contain microstimulation.

\section{Effects of microstimulation on psychophysical performance}

Microstimulation induces a shift in the psychometric function. We tested the hypothesis that the activity of direction selective neurons underlies perceptual judgements of motion direction by applying electrical microstimulation to clusters of directionselective neurons while a monkey performed the direction discrimination task described in Materials and Methods. Successful activation of neurons encoding a similar direction of motion should cause a monkey to report that motion was in the neurons' preferred direction. In other words, a monkey should make more preferred decisions in the stimulated condition as compared to the nonstimulated condition. Four experiments in which microstimulation caused such an effect are shown in Figure 4. The psychometric functions displayed were derived from best-fitting solutions to Equation 3 (see Materials and Methods). Thus, the shapes of the stimulated and nonstimulated functions were constrained to be identical. Trials that did not contain microstimulation are shown with open symbols, and trials in which we applied microstimulation are indicated with solid symbols. At nearly every correlation level within these experiments, the monkeys made more preferred decisions when microstimulation accompanied the presentation of the visual stimulus. Figure $4 A$ shows a small effect of microstimulation in monkey $\mathrm{J}$, and Figure $4 B$ depicts a medium-sized effect in monkey E. Larger effects of microstimulation are shown in Figure $4 C$ from monkey $\mathrm{E}$ and Figure $4 D$ from monkey $\mathrm{J}$. The magnitude of the microstimulation effect is particularly striking in Figure $4 D$. At a correlation of $18.1 \%$ with motion in the neurons' null direction, the monkey made about $15 \%$ preferred decisions on nonstimulated trials. In other words, the monkey made $85 \%$ correct choices. The application of microstimulation, however, caused the monkey to make $60 \%$ preferred decisions in this condition, and thus only $40 \%$ correct choices. Microstimulation can therefore have very large effects on the monkeys' choice behavior.

To quantify the behavioral effect, we measured the horizontal 

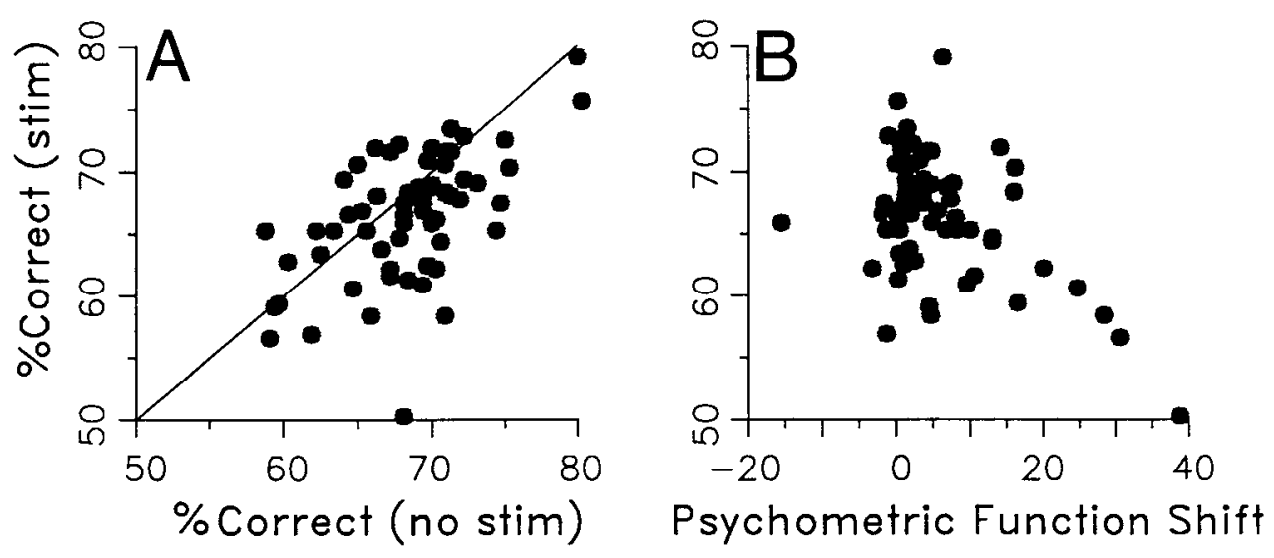

Figure 5. The effect of microstimulation on the monkey's reward rate. $A$, Percentage of correct choices on nonstimulated trials in each experiment scattered against the percentage of correct choices on stimulated trials. $B$, The percentage correct on stimulated trials plotted as a function of the shift of the psychometric function. distance between the stimulated and nonstimulated psychometric functions. This quantity is equal to $\beta_{1} / \beta_{2}$ in Equation 3 . This method of measurement quantifies the microstimulation effect in units of the visual stimulus. In other words, the shift of the psychometric function along the abscissa corresponds to the percentage of correlated dots that, if added to the visual stimulus in the neurons' preferred direction, would mimic the behavioral effect of microstimulation. We will henceforth refer to this shift as the "equivalent visual stimulus" of the microstimulation effect. The equivalent visual stimuli for the four experiments depicted in Figure 4 were $5.6 \%$ correlated dots for Figure $4 A, 10.0 \%$ for Figure $4 B, 14.2 \%$ for Figure $4 C$, and $30.6 \%$ for Figure $4 D$. Psychophysical threshold in these experiments was typically between $5 \%$ and $20 \%$ correlation. Thus, the equivalent visual stimulus provided by electrical microstimulation of MT can be substantially larger than the actual visual stimuli required to support threshold performance.

Note that the monkeys were always rewarded for making a correct choice and therefore had no incentive in terms of reward rate to make an excess of decisions in either direction. In fact, the monkeys frequently received fewer rewards on stimulated trials than on nonstimulated trials. Figure $5 A$ shows a scatterplot of percentage correct choices on nonstimulated trials versus percentage correct on stimulated trials for the 62 experiments. In general, reward rates were between $60 \%$ and $75 \%$ correct, as intended (see Materials and Methods). For many experiments, the reward rate was similar on stimulated and nonstimulated trials. However, more of the data points lie below the diagonal than above it. On average, the monkeys made significantly fewer correct choices on stimulated trials $(66 \%$ correct) than on nonstimulated trials (68\% correct; $p=0.001$, paired $t$ test). Moreover, the monkeys tended to make fewer correct choices as the microstimulation effect became larger. Figure $5 B$ shows the percentage correct on stimulated trials as a function of the magnitude of the stimulation-induced shift of the psychometric function. As the size of the shift increased, the percentage correct on stimulated trials decreased $(r=-0.479 ; p \leq 0.0001)$. From these data, it is clear that behavioral effects such as those in Figure 4 occurred despite a decrease in overall reward rate.

The equivalent visual stimulus for each of the 62 experiments is shown in Figure $6 \mathrm{~A}$. Positive values on the abscissa indicate leftward shifts of the psychometric function, or increases in preferred decisions. Negative values correspond to rightward shifts of the psychometric function. If microstimulation had no effect on the monkey's performance, we would expect this distribution to be centered about zero. The distribution, however, is shifted to the right of zero, indicating that microstimulation, on average, increased the proportion of decisions in favor of the neurons' preferred direction. In 30 of the 62 experiments, microstimulation had a statistically significant effect $(p<0.05)$ on the monkey's choice bias, and these experiments are indicated with open bars. In 29 of these 30 significant experiments, microstimulation resulted in a leftward shift of the psychometric function. The same result is evident in the expanded data set in Figure $6 B$ (see Materials and Methods). We obtained significant effects in 89 out of 139 experiments (64\%), and microstimulation resulted in an increase in preferred decisions in 86 out of the 89 experiments $(97 \%)$. The direction of the behavioral effect could be predicted, therefore, from the physiological properties of the neurons at the stimulation site. These data support the basic notion that microstimulation cnhanced the intracortical signal representing motion in the neurons' preferred direction.

In three experiments, microstimulation resulted in a significant rightward shift of the psychometric function (negative values, Fig. 6B). This rightward shift may have resulted from activation of neurons whose preferred direction was opposite to that measured at the stimulation site. Since adjacent columns in MT frequently have opposite preferred directions (Albright et al., 1984), and since we were uncertain concerning the precise arrangement of direction columns near the electrode tip (see Materials and Methods and Discussion), the activation of neurons with opposite preferred direction to those at the stimulation site is a plausible explanation for these data. In fact, we are somewhat surprised that this phenomenon did not occur more often.

Effects on slope of the psychometric function. To cxamine the effect of microstimulation on the shape of the psychometric function, we refit data from each of the 62 experiments with Equation 2, thus allowing the shape of the stimulated psychometric function to differ from the nonstimulated psychometric function. We found that microstimulation had a significant effect on shape in a minority of experiments ( 12 out of 62 experiments; $\beta_{3}$ significantly different from $0, p<0.05$ ). Eight of these changes were accompanied by a significant shift in the psychometric function. Figure 7 summarizes these data by showing the change in slope of the stimulated psychometric function for each experiment. This value was derived by subtracting the slope of the nonstimulated psychometric function from the slope of the stimulated psychometric function at the point on each curve where the slope is steepest $\left[\left(\beta_{2}+\beta_{3}\right) / 4-\beta_{2} / 4\right]$; for our model this is always the $50 \%$ preferred decision point. Negative values 


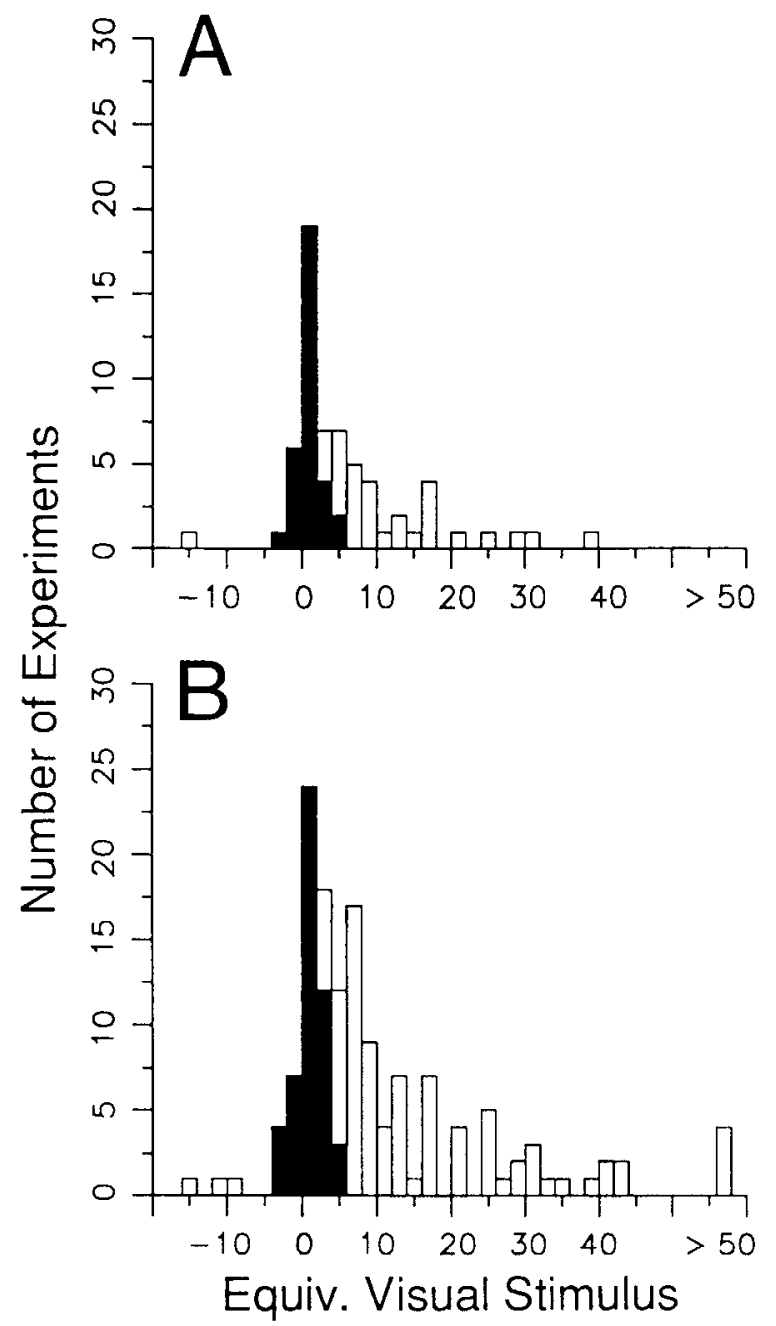

Figure 6. Frequency histograms showing for each experiment the equivalent visual stimulus of the behavioral effect of microstimulation. Positive values on the abscissa indicate leftward shifts of the stimulated psychometric function, or increases in preferred decisions. Negative values correspond to rightward shifts of the psychometric function, or decreases in preferred decisions. The open bars represent experiments in which microstimulation significantly biased the monkeys' perceptual choices ( $p<0.05$, logistic regression analysis). $A$, Results of the original 62 experiments conducted in three monkeys. Significant effects of microstimulation were obtained in each monkey (18 significant out of 38 in monkey $J, 9$ out of 16 in monkey $\mathrm{E}$, and 3 out of 8 in monkey L). $B$, Histogram showing the shift in the stimulated psychometric function in the expanded data set representing 139 experiments in the same three monkeys (see Materials and Methods). This histogram includes the data presented separately in $A$.

on the abscissa reflect slopes that have become flatter due to microstimulation. Positive values reflect steepened slopes. The open bars represent the experiments in which the slope change was statistically significant $(p<0.05)$. In 10 of the 12 experiments with significant effects, microstimulation flattened the slope of the psychometric function. In the remaining two experiments, microstimulation steepened the psychometric function.

The slope measurements illustrated in Figure 7 may have been inaccurate in some experiments because we collected data at a restricted range of correlation levels comprising only a part of the psychometric function. Consequently, we conducted 46

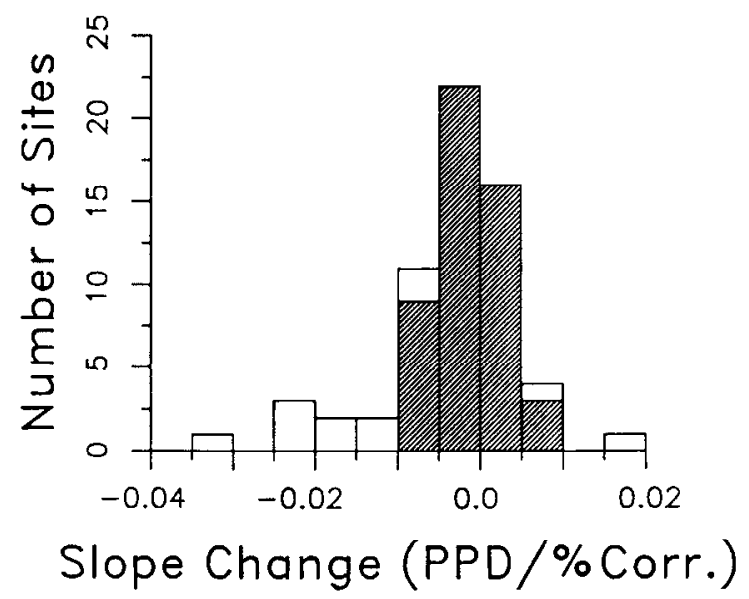

Figure 7. Frequency histogram showing the stimulation induced change in slope at the steepest point of the psychometric function. For each site tested, the slope change is indicated as the change in proportion preferred decisions (PPD) made per percentage correlation. Positive values on the abscissa correspond to slopes that were steeper for stimulated psychometric functions than for nonstimulated psychometric functions. Negative values indicate that microstimulation flattened the slope of the psychometric function. The open bars indicate experiments in which the change in slope was statistically significant $(p<0.05$, logistic regression analysis).

additional microstimulation experiments in which data were collected over the full range of correlation levels, including 100\% correlated dots - the strongest motion signal achievable by our stimulus. The results of four of these experiments are shown in Figure 8 . The scale of the abscissa of these graphs is different from those in previous figures. In Figure $8 A$, microstimulation resulted in a large shift of the psychometric function and also caused a significant slope change. Figure $8 B$ depicts the largest microstimulation effect that we have observed in terms of shift of the psychometric function. The equivalent visual stimulus of this effect was greater than $100 \%$ correlated dots. In addition, microstimulation caused a significant slope change (flattening) in this experiment. In the experiment shown in Figure $8 \mathrm{C}$, microstimulation shifted the psychometric function without changing the slope, demonstrating that large shifts can occur in the absence of slope changes. Finally, Figure $8 D$ represents an experiment in which microstimulation caused a significant steepening of the psychometric function.

Figure 9 illustrates the slope change observed in the 46 additional experiments conducted in monkey $\mathbf{J}$ with correlation levels ranging from $0 \%$ to $100 \%$. The abscissa is identical to that shown in Figure 7 . We observed 10 significant effects on slope in these experiments, and the slope of the psychometric function was flattened in eight of those. These data indicate that the tendency to flatten the psychometric functions did not result from the restricted range of correlations tested in the original 62 experiments. Instead, the flattened slope of the stimulated psychometric function suggests that microstimulation added noise to the neural processes underlying perceptual judgements. A likely source of noise is current spread to other columns encoding different dircetions of motic ?.

Time course of the microstimulation effects. We frequently noticed that the strength of the microstimulation effect seemed to wane over the $1 \mathrm{hr}$ course of an experiment. We analyzed the time course of this effect by calculating the proportion of 

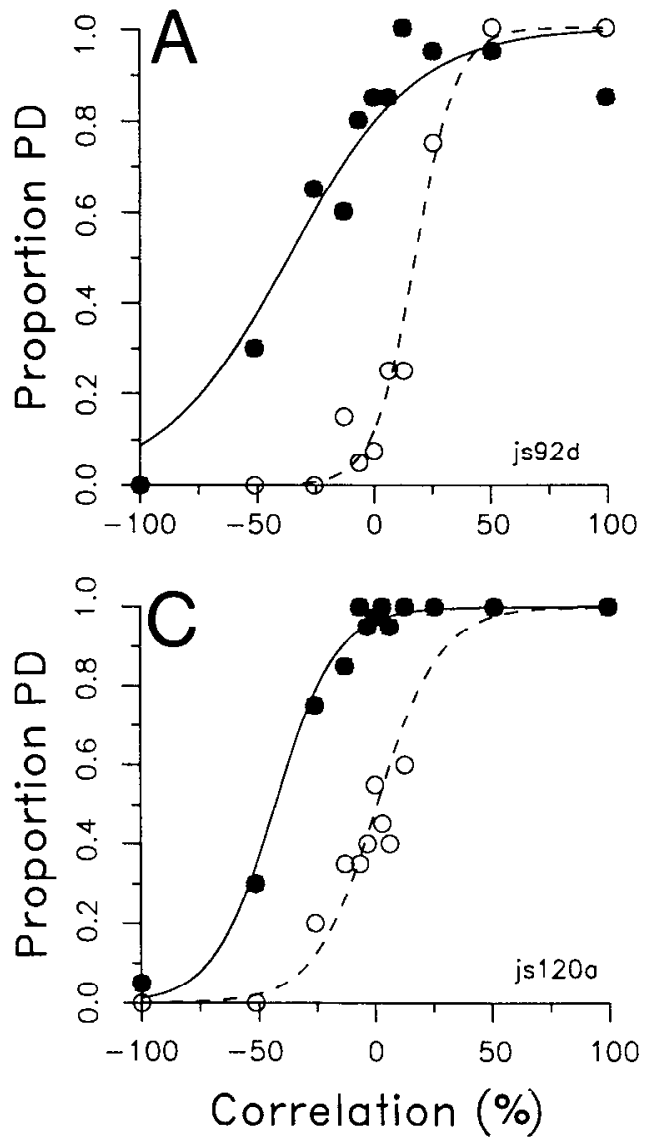
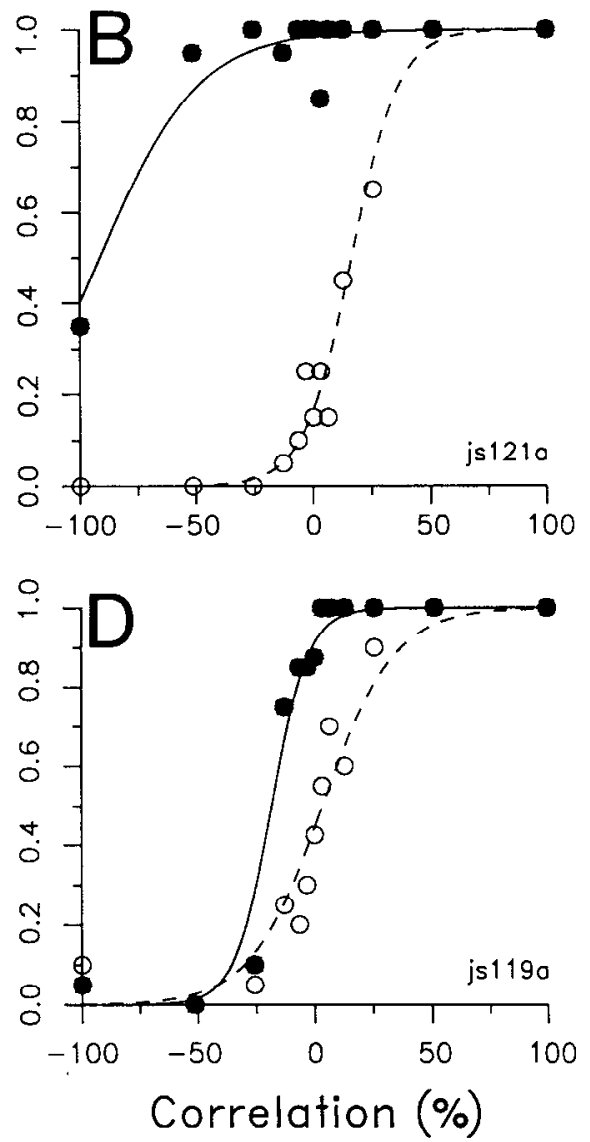

Figure 8. The effect of microstimulation at four stimulation sites when the correlation levels tested ranged from $0 \%$ correlation to $100 \%$ correlation. These data were fit with Equation 2, in which the shapes of the stimulated and nonstimulated psychometric functions were free to differ. Note the change in scale on the abscissa from previous figures. $A$, A large effect of microstimulation in which the slope of the psychometric function was also significantly flattened $(p \leq 0.0001$, logistic regression analysis). $B$, The largest microstimulation effect we have observed in terms of the shift in the psychometric function. The cquivalent visual stimulus of the effect of microstimulation in this experiment was $>100 \%$ correlated dots. The slope of the psychometric function was also significantly flattened $(p \leq 0.0001)$. $C$, A large effect of microstimulation with no effect on the slope of the psychometric function. $D$, An experiment in which the slope of the psychometric function was significantly steepened by microstimulation $(p \leq 0.0001)$. preferred decisions made by the monkey as a function of the trial number within an experiment. We then pooled the data across the 30 experiments with statistically significant effects (open bars, Fig. 6A). Since microstimulation usually had its largest effects at low correlation levels, the time course of the effect is more easily observed at low correlations. We therefore restricted the analysis to correlation levels at which the monkeys' percentage of correct choices was not significantly different from random performance on nonstimulated trials $\left(\chi^{2}\right.$ test, $p>$ $0.1)$. In effect, this requirement meant that data were included only for $0 \%$ correlation and other low correlation levels. The result of this analysis is shown in Figure 10. The $\times s$ in Figure 10 correspond to stimulated trials, and the circles indicate nonstimulated trials. Each point summarizes the data within a bin of 10 trials.

This analysis shows that the effect of microstimulation is greatest during the first 100 trials in an experiment. During this interval, the monkeys made preferred decisions on more than $70 \%$ of stimulated trials $(\times$ s, Fig. 10$)$. On nonstimulated trials during the same interval, the monkeys rapidly developed a small choice bias toward the direction opposite to the neurons' preferred direction, as indicated by the circles from trial 10 onward where the monkeys made only $35-45 \%$ preferred decisions. This choice bias toward the neurons' null direction will be discussed in section III of the Results.

As the experiments progressed, the effect of microstimulation became gradually weaker. The proportion preferred decisions made on stimulated trials decreased, and correspondingly, the proportion preferred decisions on nonstimulated trials in- creased. Multiunit recording after a microstimulation experiment usually revealed that the responses of neurons at the electrode tip were decreased. The reduced responsiveness could have been caused by neuronal death or fatigue, by small changes in electrode position during the experiment, or by electrode etching caused by the positive current pulses. We will address these issues in detail in the Discussion.

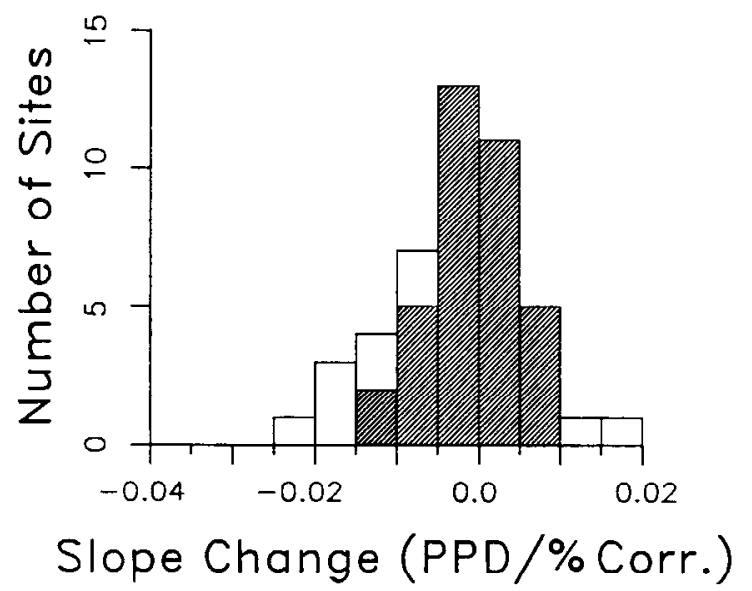

Figure 9. Frequency histogram showing the change in slope of the psychometric function for the 46 experiments in which we tested the effects of microstimulation at correlation levels ranging from $0 \%$ to $100 \%$. The axes are identical to those described in Figure 7 . Open bars represent experiments in which microstimulation had a significant effect on the slope $(p<0.05)$. 


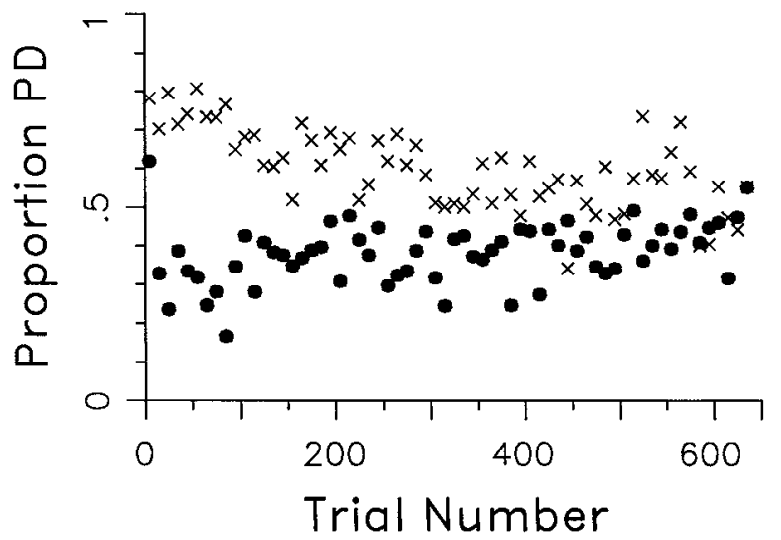

Figure 10. Time course of the microstimulation effect in the 30 experiments in which microstimulation significantly biased the monkeys' choices. The proportion preferred decisions $(P D)$ made by the monkeys is plotted as a function of trial order within an experiment. The $\times s$ represent stimulated trials; the circles indicate nonstimulated trials. Each data point represents the proportion preferred decisions within a 10 trial bin, averaged across all experiments. This analysis includes only trials complcted at correlation levels for which the monkey performed at chance levels on nonstimulated trials $\left(p>0.1, \chi^{2}\right.$ test).

Laminar location of stimulation sites. Due to the posterior approach to MT employed in these experiments, our electrodes entered MT though the infragranular layers in the posterior bank of the STS. Since MT is a folded structure lying in the depths of the STS, penetrations traversed MT tangentially on some occasions and almost perpendicularly on others, depending upon the exact points of entry and exit. We were therefore uncertain as to the laminar location of most of our stimulation sites. Despite this ambiguity, we gained a distinct impression over the course of the study that microstimulation effects could be obtained in any lamina. We have several examples of significant effects when the stimulation site was located just after the electrode entered MT (almost certainly layer 6 or layer 5), and we have observed effects of microstimulation at stimulation sites found just before the electrode exited MT into the lumen of the superior temporal sulcus (almost certainly superficial layers). In monkey $\mathrm{J}$, we attempted to gather precise information concerning the location of stimulation sites yielding very large effects (shifts $>20 \%$ ). During the last 4 weeks of the animal's life, we placed 11 electrolytic lesions in one MT marking the location of large-effect sites. Histological reconstruction of electrode penetrations allowed us to identify eight of the lesions with certainty. Six of the eight lesions were associated with layer 4 . Four of thesc six were within layer 4 , one was on the border between layers 3 and 4, and another was on the border between layers 4 and 5 . One of the remaining two lesions was located in lower layer 3, and the last lesion was found at the boundary between layers 5 and 6 . It appears, therefore, that large-effect experiments are obtained more frequently in or near layer 4.

\section{Mechanism of the microstimulation effect}

The directionally specific effect of microstimulation suggests that the stimulating current preferentially affects sensory signals that encode a particular direction of motion. Alternatively, the
Figure 11. An experiment in which the topographic specificity of the behavioral effect of microstimulation was tested. $A$, A diagram showing the fixation point $(F P)$ for the monkey, receptive ficld of neurons at the stimulation site (shaded circle), preferred direction of the neurons (arrow), and the location of the visual stimulus aperture when it was remote from the neurons' receptive field (large open circle). The distance from the center of the neuronal receptive field to the center of the remote aperture was 2.4 receptive field diameters. See text for details of the experimental methods. $B$, Effect of microstimulation when the visual stimulus was presented over the neurons' receptive field. Axes are the same as in Figure 4. $C$, Effect of microstimulation when the visual stimulus was presented at the remote location. Each data point was based upon 25 trials, except for $0 \%$ correlation, where 50 trials were conducted.

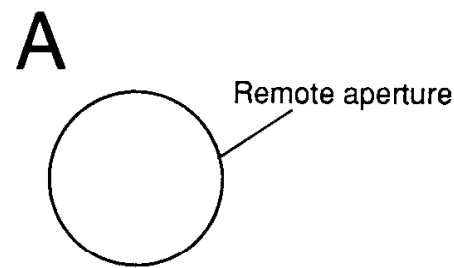

FP<smiles>CC(C)(C)[SiH3]</smiles>

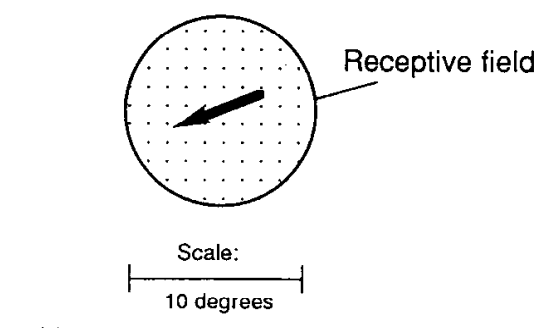

es73a
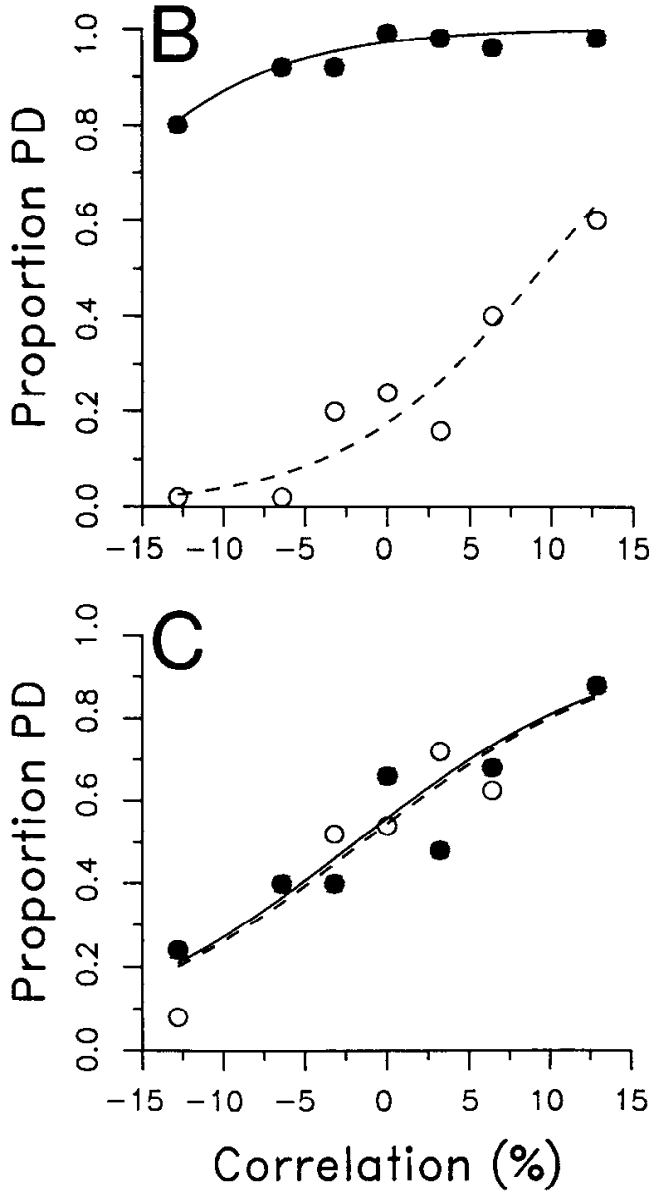

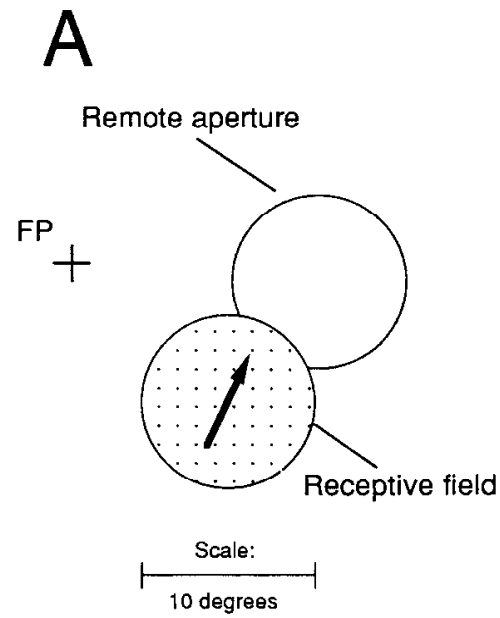

es77a
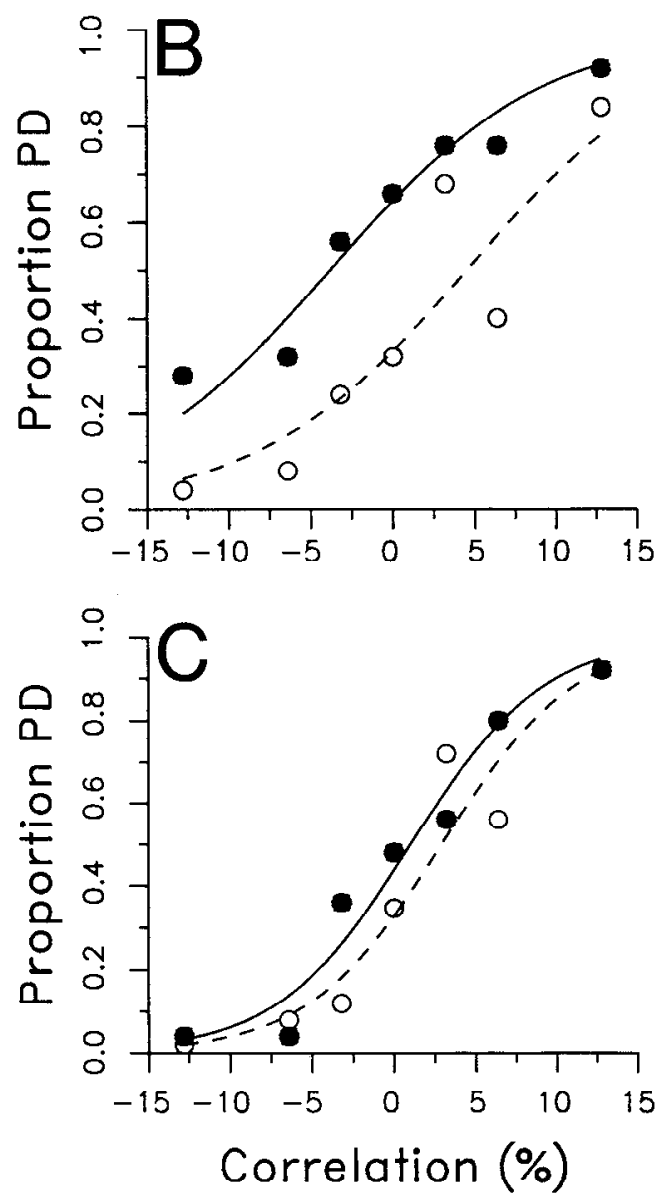

Figure 12. Anothcr experiment testing the topographic specificity of the behavioral effect. $A$, A diagram displaying the conditions of the experiment. All labels are the same as in Figure 11. The remote visual stimulus aperture abutted the neurons' receptive field. $B$, The effect of microstimulation when the monkey discriminated the direction of motion at the receptive field location. $C$, The effect of microstimulation on discriminations at the remote location. microstimulation effect might result from a direct influence on either (1) circuits involved in decisional processes or (2) motor circuits involved in planning and executing saccadic eye movements, the opcrant responsc on our task. We will consider both of these possibilities further in the Discussion. Here, we present data suggesting that microstimulation does not affect one possible type of decisional mechanism and that microstimulation does not affect motor circuits directly.

Decisional mechanisms. Although we do not know where or how perceptual decisions are implemented in the cerebral cortex, it is possible that microstimulation could have affected neuronal circuits that influence what is decided without influencing what is seen. At least two types of mechanisms underlying decisional processes are conceivable. First, a decisional mechanism might be topographically restricted such that different neuronal circuitry is invoked depending upon the spatial location of the visual stimulus being discriminated. Alternatively, the decisional mechanism might be generalized in the sense that the same neuronal circuitry would operate regardless of visual stimulus location. If microstimulation in MT dircctly affects a generalized decisional mechanism utilized in our task, we would expect the monkey's choices to be biased regardless of the visual stimulus location. If, on the other hand, microstimulation exerts its primary influence on sensory processes or on topographically restricted decisional mechanisms, we would only obtain effects of microstimulation at locations near the receptive field of the stimulated neurons.

We therefore designed an experiment to test the topographic specificity of the microstimulation effects; we will subsequently refer to this as the "spatial offset" experiment. On half of the trials in this experiment, the monkey discriminated the direction of motion in a visual stimulus placed remotely from the neurons' receptive field, and on the other half of the trials, the monkey discriminated the direction of motion in a visual stimulus at the receptive field location. The visual stimulus was presented at the same absolute spatial location for both the remote and receptive field conditions, but the retinotopic location of the visual stimulus was alternated from trial to trial by repositioning the fixation point. As in the previous experiments, the axis of motion, the speed of the correlated motion signal, and the size of the stimulus aperture were chosen to match the preferences of neurons at the stimulation site. As before, trials were conducted at three correlation levels near threshold as well as $0 \%$ correlation. On half the trials at each spatial location, we applied microstimulation (same parameters as before) to stimulation sites selected in the usual manner (see Materials and Methods). All trial conditions were randomly interleaved, and the monkey was rewarded only for correct choices. The location of the remote aperture was chosen to be at a retinal eccentricity comparable to that of the receptive field aperture. Psychophysical thresholds were therefore approximately equal at the two locations.

Figure $11 \mathrm{~A}$ depicts the psychophysical conditions of a spatial offset experiment in which the remote visual stimulus location was in the upper visual field (large open circle) while the receptive field of neurons at the stimulation site was in the lower field 
Figure 13. An experiment testing the effects of microstimulation when the remote visual stimulus aperture partially overlapped the neurons' receptive field. $A$, A diagram of the experimental conditions. The remote visual stimulus aperture overlapped the neurons' receptive field by half a receptive field diameter. See Figure 11 for interpretation of the labels. $B$, Effect of microstimulation when the visual stimulus aperture completely overlapped the neurons' receptive field. $C$, Effect of microstimulation in the partial overlap condition.
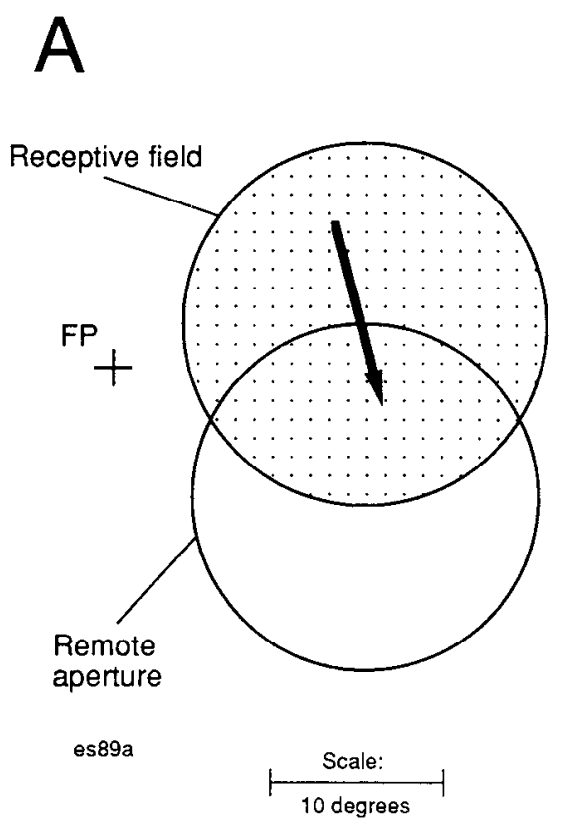
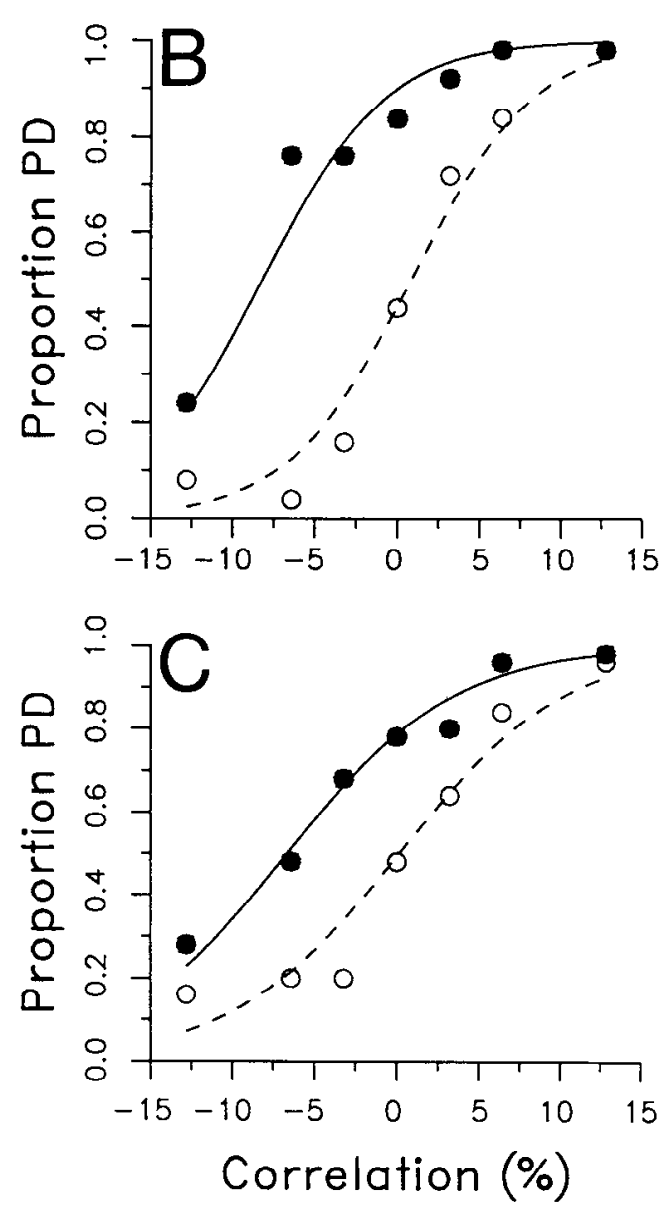

(shaded circle). For convenience of presentation, the location of the visual stimulus is shown in retinotopic coordinates, not in absolute spatial coordinates. Figure $11 B$ shows that microstimulation shifted the psychometric function by $30.0 \%$ correlated dots when the monkey performed the task for stimuli at the receptive field location. However, microstimulation had no effect when the monkey performed the task for stimuli at the remote location (Fig. $11 C$; left shift $=0.45 \%$ correlated dots, $p$ $>0.2$ ). Thus the effect of microstimulation was topographically specific: the same stimulating current that was demonstrably effective for visual stimuli at the receptive field location was completely ineffective for visual stimuli at the remote location. A similar result is shown in Figure 12. Here the remote aperture was positioned so that it abutted the receptive field of neurons at the stimulation site (Fig. 12A). Microstimulation resulted in a significant shift of the psychometric function at the receptive field location $(8.3 \%$ correlated dots, $p \leq 0.0001$; Fig. $12 B)$, but yielded a nonsignificant shift at the remote location $(1.9 \%$ correlated dots, $p>0.05$; Fig. $12 C$ ). When the remote stimulus aperture partially overlapped the neurons' receptive field, however, microstimulation typically influenced the monkey's choices for visual stimuli at the remote location, though by a smaller amount than in the case of full overlap. In Figure 13, the remote aperture overlapped the receptive field by one half the receptive field diameter. At the receptive field location, microstimulation shifted the psychometric function to the left by $9.0 \%$ correlated $\operatorname{dots}(p \leq 0.0001 ;$ Fig. $13 B)$. At the remote location, microstimulation resulted in a left shift of $6.8 \%$ correlated dots $(p \leq 0.0001$; Fig. 13C).
We conducted the spatial offset experiment 31 times in monkey E, and the results are summarized in Figure 14. The mean equivalent visual stimulus, or shift of the psychometric function, is plotted as a function of the visual stimulus location expressed as the distance, in receptive field diameters, between the center of the receptive field and the center of the remote stimulus aperture. When the visual stimulus aperture was directly over the neurons' receptive field, microstimulation on average induced a left shift of $12.4 \%$ correlated dots (center-to-center distance $=0$; Fig. 14). This data point is derived from one-half of the trials in each of the 31 experiments. When the remote aperture overlapped the receptive field by half a diameter (13 experiments), microstimulation on average produced a left shift of $7.9 \%$ correlated dots (center-to-center distance $=0.5$ ). This effect was significantly smaller than the effect induced at the receptive field location in the same experiments $(p<0.01$, paired $t$ test). For remote apertures separated from the receptive field by one or more receptive field diameters, there was no effect on average ( 5 experiments at center-to-center distance $=$ 1 , and 13 experiments at center-to-center distance $>1$ ). In three individual experiments at remote locations with center-to-center distances $>1$, however, microstimulation yielded small but significant shifts of the psychometric function (shifts of $6.2 \%$, $3.2 \%$, and $-3.3 \%$ correlated dots; $p<0.05$ ). Overall, these data suggest that microstimulation in MT has little effect on discrimination performance for visual stimuli remote from the neurons' receptive field. Therefore, microstimulation does not appear to affect a generalized decisional mechanism. To account for the microstimulation effects with decisional mechanisms, one must 
invoke the existence of multiple, parallel mechanisms that receive topographically localized inputs.

Microstimulation does not affect eye movements directly. We considered two possible ways in which microstimulation in MT might directly affect motor circuits controlling saccadic eye movements. First, microstimulation might cause an eye movement toward the receptive field of neurons at the stimulation site. Second, microstimulation might cause an eye movement in a direction similar to the preferred direction of neurons at the stimulation site. Several of our experiments provide evidence inconsistent with these hypotheses.

To rule out the first possibility, we exploited the fact that the preferred direction of MT neurons can change abruptly by $180^{\circ}$ as an electrode moves from one cortical column to another. An cxamplc of this type of transition is shown in Figure 15A. The left side of this figure depicts an electrode penetration through area MT, and the right side of the figure shows the receptive fields encountered at selected points in the penetration. " 0.0 mm" marks the beginning of electrode travel in MT gray matter. Approximately $0.85 \mathrm{~mm}$ into the penetration, neurons responded robustly to upward moving visual stimuli at a receptive field location shown on the right. For the next $100 \mu \mathrm{m}$ of electrode travel, neurons recorded from had a similar preferred direction and receptive field location. As the electrode advanced from $0.95 \mathrm{~mm}$ to $1.05 \mathrm{~mm}$, however, we observed an abrupt shift in preferred direction. Between $1.05 \mathrm{~mm}$ and $1.35 \mathrm{~mm}$, the neurons responded optimally to downward motion. The receptive field location for these neurons was identical to that for neurons on the other side of the transition point. We conducted microstimulation experiments on both sides of the transition point, as indicatcd by the $\times s$ that mark stimulation sites 7 and 8 . The psychophysical task in these two experiments was identical. In both cases, the monkey discriminated upward from downward motion shown at an identical location in the visual field. Moreover, the eye movements required to indicate judgements of motion direction were identical in the two experiments. The only difference between the two experiments was a $300 \mu \mathrm{m}$ separation in the position of the electrode tip.

Figure $15, B$ and $C$, shows the results of the two experiments. In both cases, microstimulation biased the monkey's choices toward the neurons' preferred direction. Thus, with the electrode placed at site 7, the monkey chose more frequently in favor of the downward direction, but with the electrode located at site 8 , the monkey made more decisions in favor of the upward direction. Since the receptive field location and the saccadic eye movements used to report decisions were the same in both experiments, it is difficult to account for the obscrvations by hypothesizing that microstimulation causes eye movements in the general direction of the receptive field. We obtained a similar result on one other occasion, biasing a monkey's choices in opposite directions when microstimulation experiments were conducted on both sides of an abrupt, $180^{\circ}$ transition in preferred direction.

The data presented in Figure 16 argue against a second variation of the motor hypothesis - that microstimulation simply causes an eye movement similar in direction to the neurons' preferred direction. We tested this notion by conducting experiments in which the directions of the eye movements to the two choice LEDs were similar. In Figure $16 \mathrm{~A}$, the eye movement that indicated a preferred choice differed by $27^{\circ}$ in angle from the eye movement that indicated a null choice. In Figure $16 \mathrm{C}$, the difference in angle between the two eye movements was only

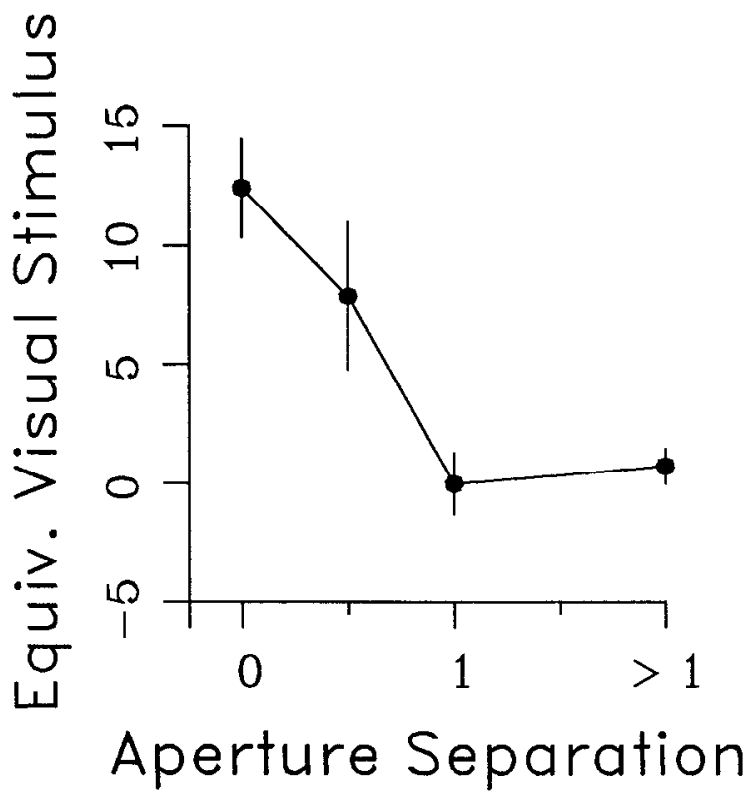

Figure 14. Summary of the effects of microstimulation for varying degrees of topographic correspondence between the stimulus aperture and the receptive field. Data were obtained in 31 experiments in monkey $\mathrm{E}$. The ordinate shows the average behavioral effect of microstimulation for each condition. The abscissa indicates the separation between the visual stimulus apcrture and the neurons' receptive field expressed as the distance (in receptive field diameters) between the center of the receptive field and the center of the stimulus aperture The error bars represent the standard error of the mean. The effect of microstimulation was largest when the visual stimulus aperture overlapped the neurons' receptive field completely (mean shift $=12.4 \%$ correlated dots). When the visual stimulus overlapped the receptive field by one-half a receptive field diameter ( 13 experiments), the mean shift was smaller $(7.9 \%$ correlated dots). However, when the visual stimulus aperture either abutted the neurons' receptive field ( 5 experiments) or was more distant from the receptive field (13 experiments), microstimulation on average had little effect on the monkey's perceptual performance (abutting, mean shift $=-0.02 \%$ correlated dots; more distant, mean shift $=0.7 \%$ correlated dots).

$13^{\circ}$. In these experiments, the eye movements that indicated preferred and null choices differed primarily in amplitude, not in direction. Despite the similarity of eye movement angles, microstimulation biased the monkey's choices toward the neurons' preferred direction in both experiments (Fig. 16B,D). The experiment illustrated in Figure $16, A$ and $B$, provides a compelling counterexample to the hypothesis that microstimulation causes an eye movement in a similar direction to the neurons' preferred direction. In this experiment, the preferred direction of the stimulated neurons was to the left and slightly upward, but the eye movement that indicated a preferred choice was to the right and slightly downward-opposite to the preferred direction of the neurons. These data demonstrate that our results cannot be explained by postulating that microstimulation generates saccades in a direction similar to the preferred direction of the stimulated neurons.

\section{Behavioral effect on nonstimulated trials}

Null choice bias. Early in this study, we noticed that the monkeys tended to have a choice bias toward the neurons' null direction on nonstimulated trials (e.g., Figs. $4 A-D, 8 A-D$ ). At $0 \%$ correlation, where there is no net motion signal contained in the 
Figure 15. Two experiments in monkey $\mathrm{L}$ conducted at stimulation sites 300 $\mu \mathrm{m}$ apart with opposite preferred directions. $A$, Schematic diagram of an electrode penetration through MT (left side) and the receptive fields encountered at selected points along the penetration (right side: FP, fixation point; circle, receptive field boundary; arrow, preferred direction). Two patches of cortex are marked with braces. The physiological properties of the two patches were similar, except that the preferred directions were $180^{\circ}$ opposed. We conducted microstimulation experiments on both sides of the transition point (site 7 and site 8 , marked by the $\times s$ ). The psychophysical task was identical in the two experiments in every respect except for the location of the electrode tip. $B$, The results of the microstimulation experiment conducted at site 8 . The axes and symbols are the same as in Figure 4. Microstimulation biased the monkeys' choices toward the neurons' preferred direction, resulting in a significant $(p=0.001)$ shift of the psychometric function of $4.2 \%$ correlated dots. $C$, The results of the microstimulation experiment conducted at site 7. Microstimulation again biased the monkey's choices toward the neurons' preferred direction (shift $=3.6 \%$ correlation; $p=0.005$ ). Note that at stimulation site 8 , microstimulation biased the monkey's choices upward, but that at stimulation site 7, the monkey's choices were biased downward.

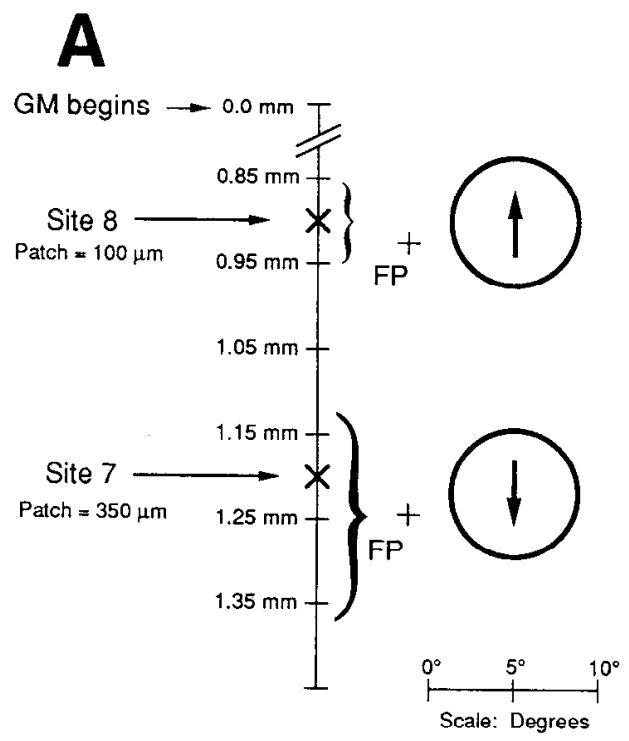

A

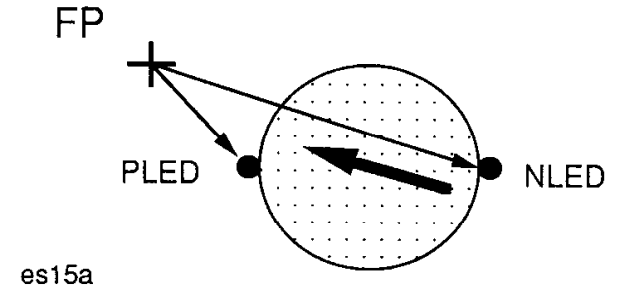

Figure 16. Two experiments in which eye movements of similar angles indicated preferred direction $(P D)$ choices and null direction choices. In these experiments, the monkey indicated its choices largely by changing the amplitude, not the angle, of its saccades. $A$, A diagram of the psychophysical conditions in one experiment showing the fixation point $(F P)$, receptive field ( $c i r-$ cle), preferred direction (arrow), and target LEDs (PLED and NLED). The difference in the angle of eye movement required to indicate choices was $27^{\circ} . B$, The results of the experiment depicted in $A$. C, Diagram of an experiment in which the eye movements differed by only $13^{\circ} . D$, The results of the experiment depicted in $C$.

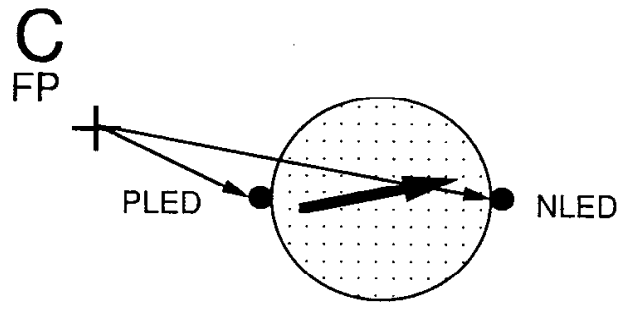

es16a

Scale:

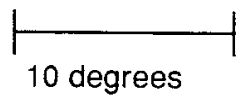

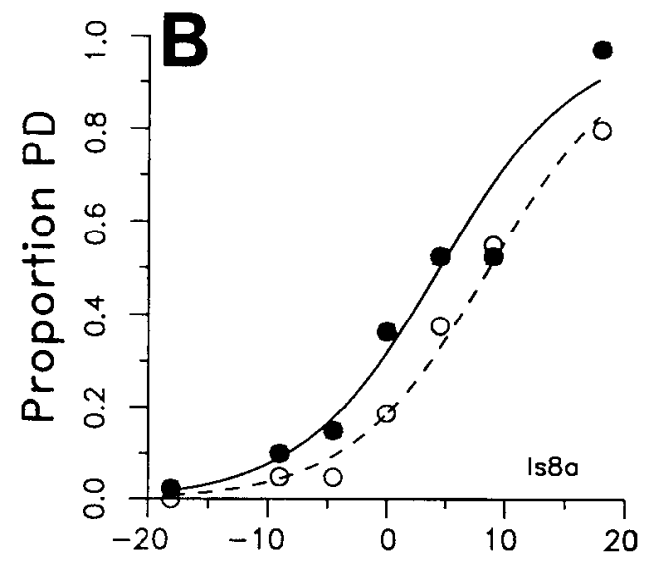
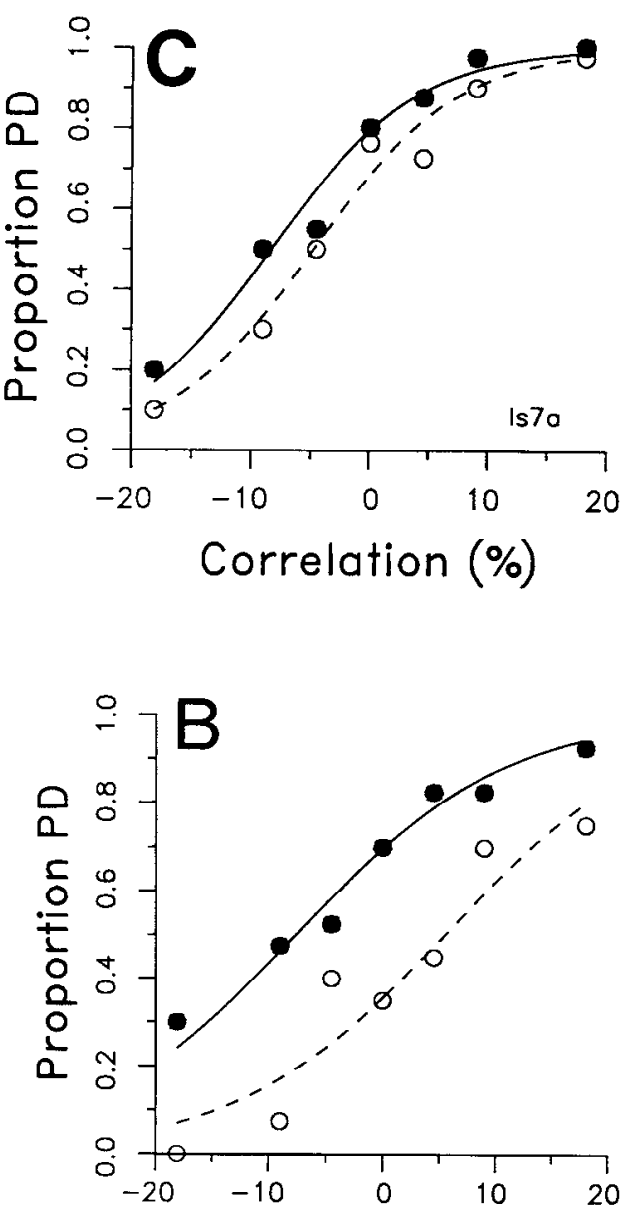

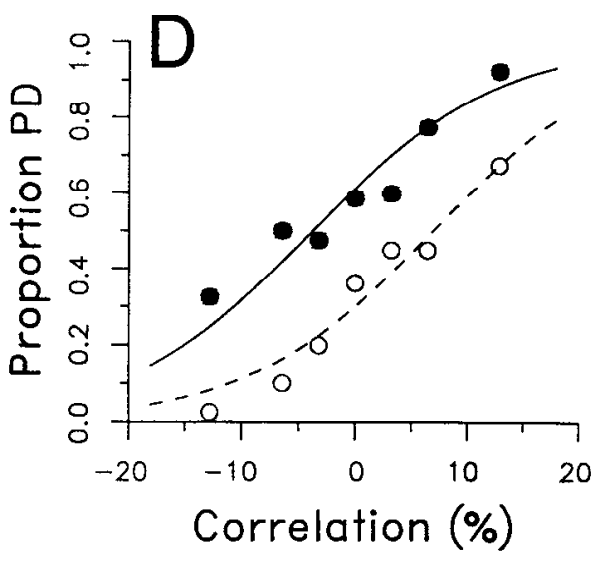



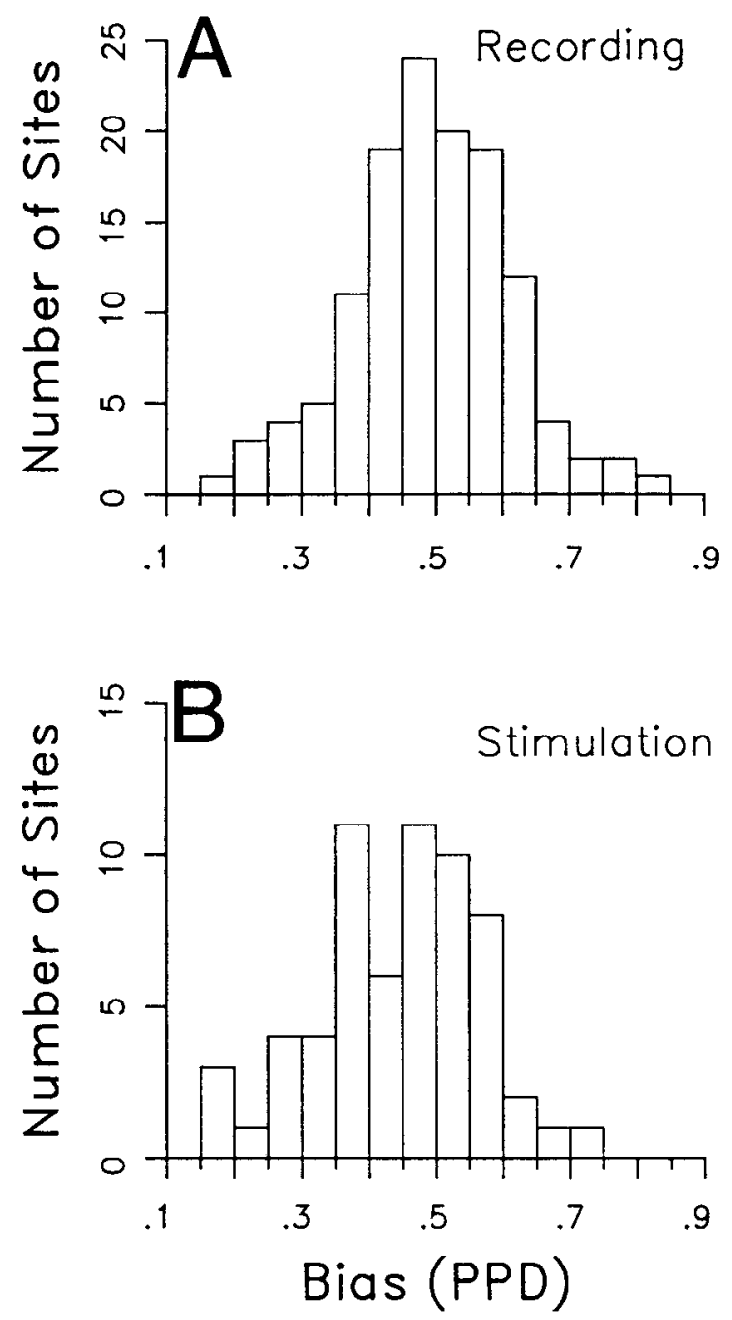

Figure 17. Frequency histograms showing the choice bias of the monkeys in physiological recording experiments and in microstimulation experiments. The intercept of the logistic regression function describing psychophysical performance [i.e., the proportion preferred decisions $(P P D)$ predicted by the fitted function at $0 \%$ correlation] is indicative of the choice bias for each experiment and is plotted on the abscissa. $A$, Distribution of choice biases for monkeys $\mathrm{J}$ and $\mathrm{E}$ obtained from 127 physiological recording experiments. No microstimulation was applicd in these expcriments. The behavioral task was otherwise similar to the one employed in the microstimulation experiments. $B$, Distribution of choice biases on nonstimulated trials for monkeys $\mathrm{J}, \mathrm{E}$, and $\mathrm{L}$ in the original 62 microstimulation experiments.

visual stimulus, we would normally expect a monkey to makc roughly $50 \%$ preferred decisions and $50 \%$ null decisions. As demonstrated in Figures 4 and 8 , this was typically not the case; the monkeys made far fewer than $50 \%$ preferred decisions on nonstimulated trials. We refer to this phenomenon as a "null choice bias," and it is apparent at correlation levels higher than $0 \%$ as well. In fact, the entire psychometric function for the nonstimulated trials frequently appears to be shifted rightward from the location one might expect.

This phenomenon was particularly puzzling because we rarely observed large choice biases during brief, qualitative inspections of the monkey's psychophysical performance prior to actual data collection. This observation suggested, paradoxically, that the null choice bias on "nonstimulated" trials was related to the presence of the stimulating pulses on "stimulated" trials. To confirm our impression that the null choice bias was a phenom-

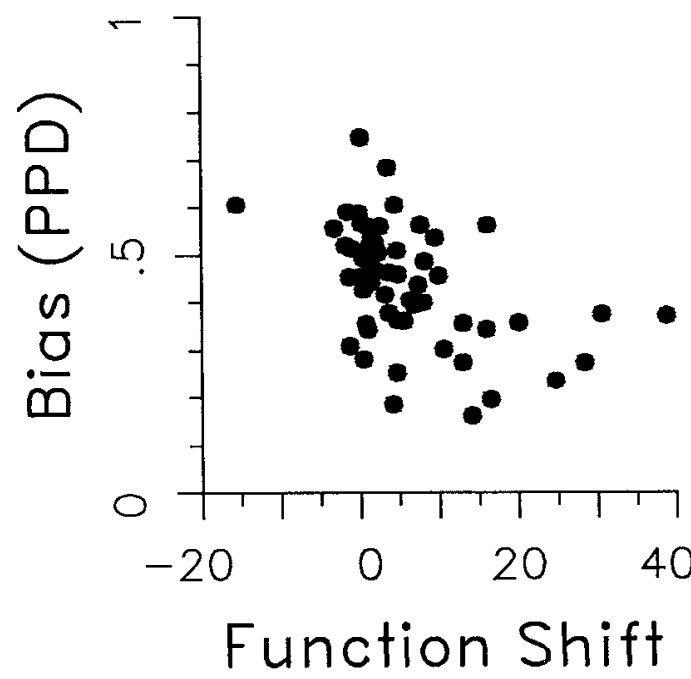

Figure 18. The relationship between the shift of the psychometric function and the choice bias on nonstimulated trials. Positive values on the abscissa correspond to leftward shifts of the psychometric function; negative values indicate rightward shifts. The ordinate plots the intercept of the nonstimulated psychometric function, which is indicative of choice bias.

enon specific to the microstimulation experiments, we compared the distribution of choice biases observed on nonstimulated trials within the present study to a distribution of choice biases obtained from the same monkeys during prior physiological recording experiments involving no microstimulation. Figure $17 A$ shows the distribution of choice biases for the recording experiments, and Figure $17 B$ illustrates similar data from nonstimulated trials in the microstimulation experiments. In each case, choice bias was measured as the y-intercept of psychometric functions like those illustrated in Figure 4. The $y$-intercept is the proportion preferred decisions expected at $0 \%$ correlation from the best-fitting solutions to Equation 3. A range of biases occurred during the physiological recording experiments, but the mean bias across the experiments was 0.492 , which was not significantly different from 0.5 ( $t$ test, $p>0.4$ ). The mean bias observed during the microstimulation experiments was 0.441 and was significantly different from 0.5 ( $t$ test, $p<0.001$ ). In addition, the means of the two choice bias distributions were significantly different from each other $(t$ test, $p$ $<0.01$ ). This comparison demonstrated that the microstimulation experiments contained an unexpected increase in the frequency of null choicc biases obscrved on nonstimulated trials. Moreover, the size of the microstimulation effects was the only aspect of our experiments predictive of the magnitude of the null choice bias on nonstimulated trials. Figure 18 shows that increases in the shift of psychometric function were proportional to increases in the null choice bias $(r=-0.477 ; p<0.0005)$.

We considered two possible mechanisms that might account for the null choice bias, the first physiological and the second behavioral. The physiological explanation holds that the null choice bias on nonstimulated trials resulted from fatiguing neurons at the electrode tip with the train of electrical pulses. By this hypothesis, neurons representing the preferred direction in a given experiment would be less active than normal on nonstimulated trials. On a $0 \%$ correlation trial, for example, the activity of neurons representing the preferred direction of motion would be less than the activity of those representing the 
Figure 19. Effects of microstimulation on a monkey's judgements of motion direction when stimulating pulses were applied just before the presentation of the visual stimulus. $A$, A schematic diagram describing the sequence of events in a trial in which microstimulation was applied during the intertrial interval. See text for methodological details. $B$, Frequency histogram showing the equivalent visual stimuli for 20 temporal offset experiments of the kind depicted in $A$. All experiments were conducted in monkey $\mathrm{E}$. The $a b$ scissa is identical to that shown in Figure 6 . The open bar represents the one experiment in which microstimulation significantly biased the monkey's choices $(p<0.05)$. $C$, Schematic diagram describing a microstimulation trial in a temporal offset experiment in which microstimulation was applied during the fixation interval. The timing of the application of stimulating pulses relative to the presentation of the visual stimulus was identical to that in $A$. This experiment differed from $A$ because we required the monkey to begin fixating prior to the application of the stimulating pulses. In all other respects, this experiment was identical to that described in $A$. $D$, Frequency histogram showing the equivalent visual stimuli for 20 temporal offset experiments of the kind described in $C$. All experiments were conducted in monkey E. The open bars denote statistically significant effects $(p<0.05)$.
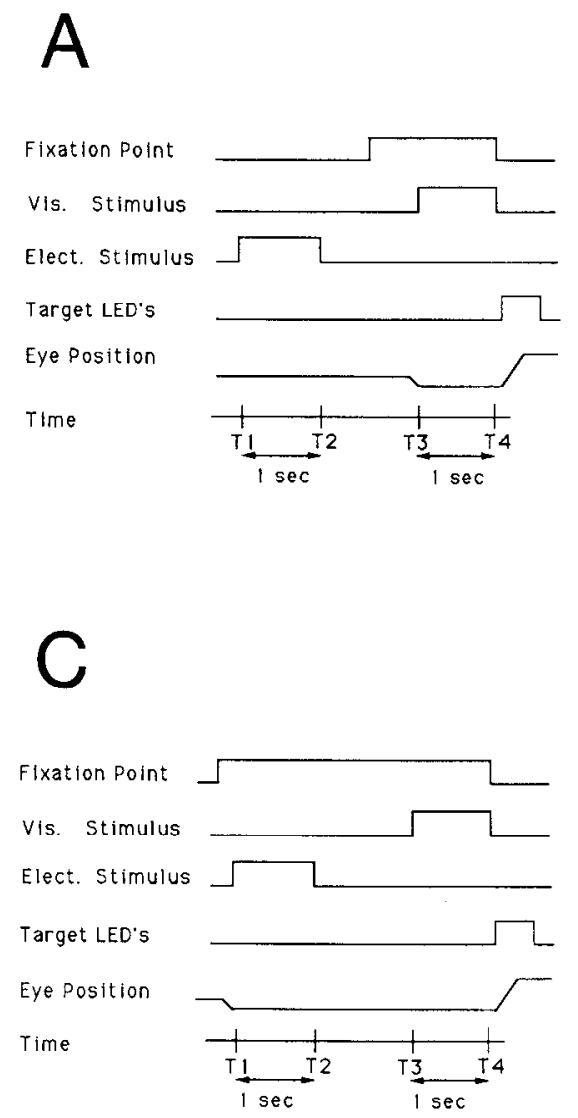

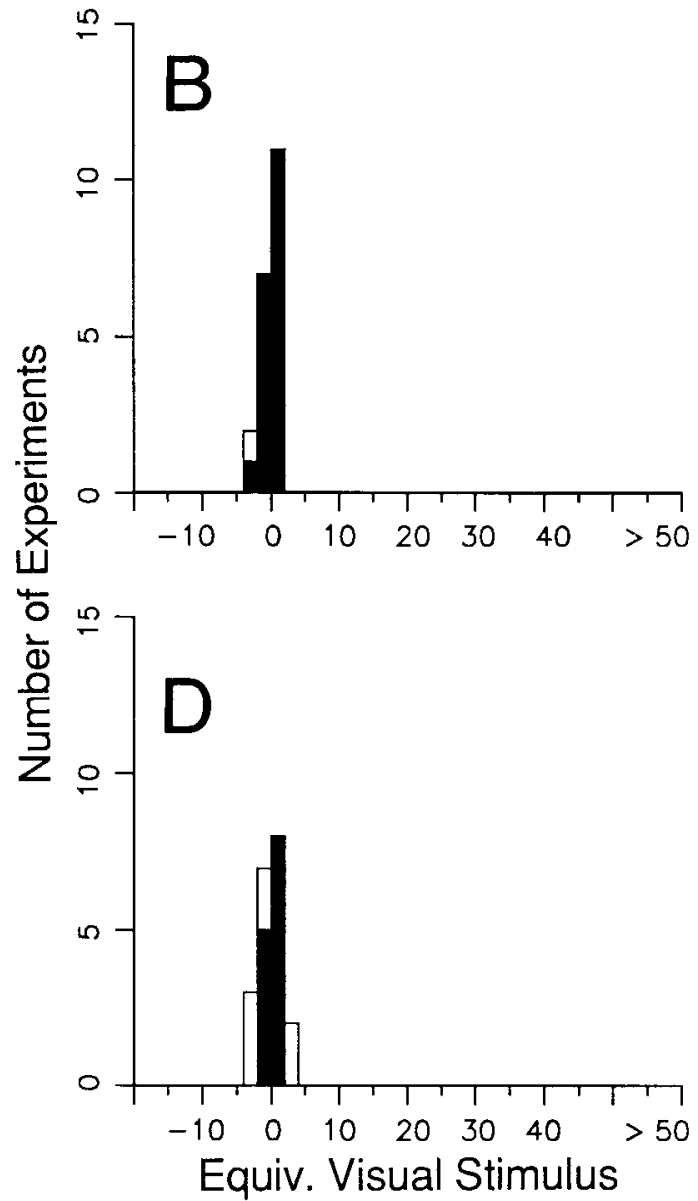

null direction, and a null decision would result. This account of the null choice bias is similar to the physiological explanation proposed to underlie the motion aftereffect, or waterfall illusion (Pantle, 1974; Vautin and Berkley, 1977; Anstis, 1980; Hammond et al., 1985, 1986; Petersen et al., 1985). In effect, the "fatigue" hypothesis implies that the null choice bias on nonstimulated trials resulted from a motion aftereffect. Alternatively, a behavioral explanation of the null choice bias maintains that the monkeys engaged in "probability matching," a behavioral strategy in which a roughly equal number of responses in favor of the preferred and null directions are made over the course of an experiment in order to match choices to the reward contingencies. In the remainder of this section, we will present the results of several experiments that together favor the behavioral interpretation over the physiological interpretation.

Temporal offset experiments. If fatigue of neurons encoding the preferred direction of motion is responsible for the null choice bias on nonstimulated trials, the effect should also be apparent if microstimulation is applied during the intertrial interval, just prior to presentation of the visual stimulus. We therefore conducted the experiment depicted schematically in Figure $19 \mathrm{~A}$. The monkey performed the direction discrimination as in previous experiments, but we applied microstimulation during the intertrial interval on half of the trials in an experiment. The other half of the trials were nonstimulated controls, and the two trial types wcre randomly interleaved. The train of electrical pulses (same parameters as before) commenced approximately $2.3 \mathrm{sec}$ before the presentation of the visual stimulus (T1, Fig. 19A) and lasted for 1 sec (until T2). The monkey viewed the visual display from time $\mathrm{T} 3$ to $\mathrm{T} 4$, and indicated directional judgements in the same manner as in previous experiments. If the null choice bias resulted from fatigue of neurons at the stimulation site, we would expect that application of microstimulation just prior to the presentation of the visual stimulus would shift the stimulated psychometric function to the right of the nonstimulated psychometric function.

We conducted this experiment 20 times in monkey $E$, and the results are shown in Figure 19B. As in Figure 6, the abscissa illustrates the shift of the psychometric function, or equivalent visual stimulus, observed in each experiment. To facilitate comparison, the scale of the abscissa in Figure $19 B$ is identical to that in Figure 6. The open bars indicate experiments in which microstimulation induced a statistically significant bias $(p<$ 0.05 ) in the monkey's choices. Overall, microstimulation had little effect on the monkey's behavior; only one experiment yielded a significant result (equivalent visual stimulus $=-2.8 \%$ correlation; $p=0.012$ ).

We were somewhat concerned that the negative result in Figure $19 B$ might be attributable in some manner to the behavioral state of the monkey. In the original experiments, microstimulation was applied while the monkey was in an attentive state, engaged in the direction discrimination task. In the experiments in Figure 19A, however, microstimulation was applied during the intertrial interval when no control was being exerted over the animal's behavior. To control for an effect of behavioral state, we conducted the experiment illustrated in Figure $19 \mathrm{C}$. 
In this experiment, the timing of microstimulation relative to the visual stimulus was identical to the experiment of Figure $19 A$, but behavioral control was added by requiring the monkey to initiate fixation before the onset of microstimulation. This experiment was performed 20 times, again with monkey $\mathrm{E}$, and the results are shown in Figure 19D. Microstimulation produced significant effects in seven experiments, but all of the effects were small ( $<4 \%$ correlation). Two of the seven significant shifts were leftward, indicating an increase in preferred decisions. The remaining significant shifts were rightward, resulting from decreases in preferred decisions. Overall, the mean of the distribution was not significantly different from 0 (mean shift $=$ $-0.186 \%$ correlated dots; $p>0.6, t$ test), indicating that microstimulation did not, on the average, shift the psychometric function toward one direction or the other. Neither distribution confirms the prediction of the "fatigue" hypothesis; microstimulation failed to induce a systematic null bias (rightward shift) on stimulated trials. These results suggest, therefore, that direct physiological effects of microstimulation do not cause the null choice bias on nonstimulated trials. Supporting this conclusion, an analysis of the original microstimulation experiments showed that the probability of making a null decision on a nonstimulated trial was not related to the interval since the last stimulated trial (data not shown). If microstimulation had caused the null choice bias directly, we would have expected the null bias to be most prominent on nonstimulated trials immediately following stimulated trials.

Probability matching. We next considered the possibility that the null choice bias on nonstimulated trials resulted from a behavioral strategy in which the monkey matches its overall frequency of preferred and null choices to the reward contingencies of the experiment. Recall that the actual direction of motion in the visual stimulus was evenly distributed between preferred and null direction in our experiments. When microstimulation caused a large excess of preferred decisions, therefore, the monkey might compensate by making an excess of null decisions on nonstimulated trials. Thus, microstimulation could shift the stimulated psychometric function to the left, and indirectly result in a shift of the nonstimulated psychometric function to the right. This explanation could also account for the correlation between the overall size of the effect and the size of the null choice bias (Fig. 18) since large null biases would be required to offset the imbalance in choice frequencies caused by large stimulation effects. This type of behavioral strategy has been studied extensively by experimental psychologists and is called probability matching because subjects make choices in a manner related to the probability of being correct (for reviews, see Estes, 1964; Atkinson et al., 1965). Note that in the context of our experiments, the monkeys can accomplish probability matching by shifting their criterion for reporting preferred direction motion. Stronger motion signals would therefore be necessary to elicit a preferred direction choice, and the overall proportion of preferred decisions within an experiment would return to a value near $50 \%$.

Data from the microstimulation experiments are consistent with the probability matching hypothesis. Figure 20 shows the choice behavior of the monkeys across the 30 statistically significant experiments as a function of the trial number in which they occurred. This analysis includes the same data illustrated in Figure 11 but pools the results for stimulated and nonstimulated trials in order to display the monkeys' overall frequency of preferred decisions during the course of the experiments.

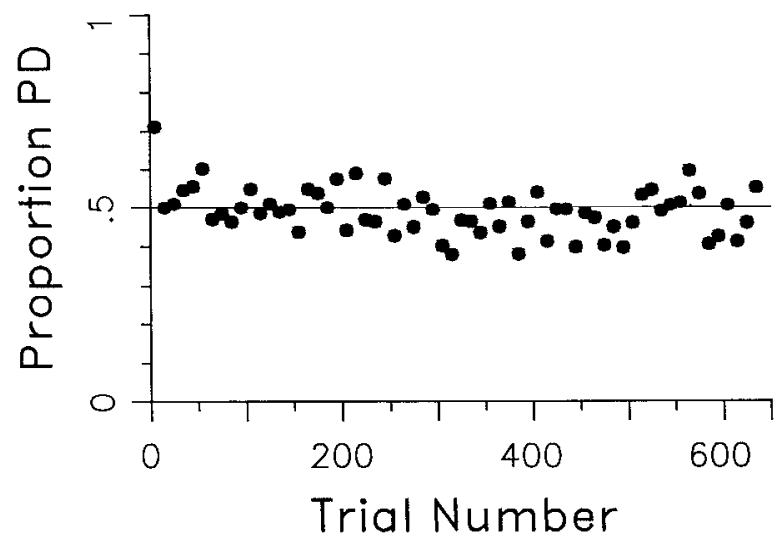

Figure 20. Time course of the monkeys' choice behavior in the 30 experiments with statistically significant effects. The proportion preferred dccisions $(P D)$ made by the monkeys is plotted as a function of trial number; each data point represents average performance across the 30 experiments. Bin size and the data included in the analysis are the same as in Figure 10. The graph pools stimulated and nonstimulated trials together and therefore comprises a combination of the separate plots in Figure 10.

Within the first 10 trials of experiments (first solid circle, Fig. 20 ), the monkeys made more than $50 \%$ preferred decisions, reflecting the influence of microstimulation on half of the trials. Very rapidly, however, the preferred choice bias disappeared, and the monkeys made approximately $50 \%$ preferred decisions (no choice bias) for the remainder of experiments. The data are therefore consistent with the central notion of probability matching - that the monkeys distribute their choices between the two possible directions of motion to match the probability of obtaining a reward in each direction.

Psychophysical reproduction of the microstimulation effect. To demonstrate that behavioral contingencies alone can account for the null choice bias, we designed a psychophysical experiment that reproduced the behavioral effect and reward contingencies of the microstimulation experiments. Specifically, psychophysical thresholds were measured exactly as before, but we mimicked the cffect of microstimulation on half of the trials in an experiment by adding to the visual stimulus a constant percentage of correlated dots in an arbitrarily defined preferred direction. Importantly, the animal was rewarded on these trials for choices that would have been correct had no motion signal been added to the visual stimulus. This strategy imitates precisely the reward contingencies on microstimulation trials in which motion signals are added to the cortical circuitry via the stimuating electrode. In the present psychophysical experiment, the "added-signal" trials were randomly interleaved with "noadded-signal" trials on which the animal was rewarded for correct choices in the usual manner. The percentage of correlated dots added to the visual stimulus in the added-signal trials was varied from experiment to experiment to mimic the range of effects observed during microstimulation experiments. Clearly, the monkey will respond to the increased preferred direction signal by making excess preferred decisions on the added-signal trials, thus shifting the psychometric function leftward relative to that for the no-added-signal trials. The key question, however, is whether the monkey will make excess null decisions (null choice bias) on the no-added-signal trials in order to maintain approximately equal choice frequencies in each direction. Prior to each experiment, we obtained normal psychophysical data 

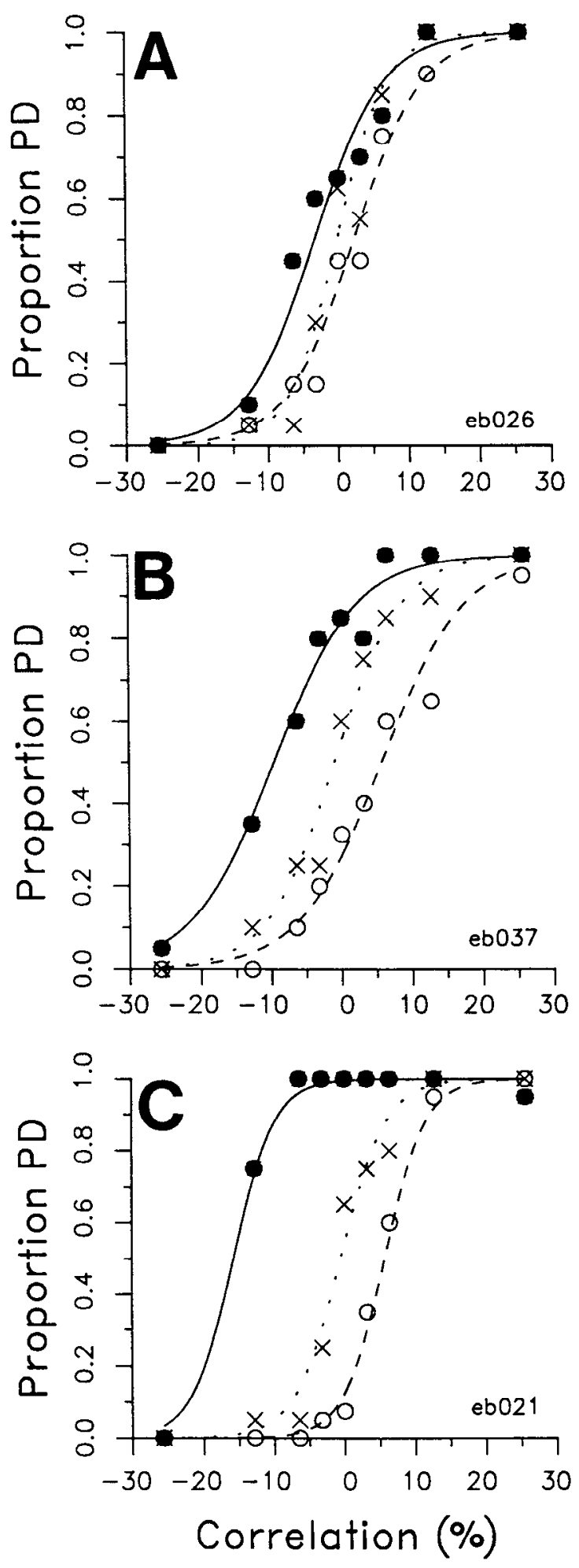

Figure 21. The effect of adding a fixed percentage of correlated dots to half the trials in three psychophysical experiments in monkey $\mathrm{E}$. The open symbols correspond to trials in which we did not manipulate the visual stimulus ("no-added-signal" trials). The solid symbols represent trials in which we manipulated the visual stimulus ("added-signal" trials). The abscissa corresponds to that described in Figure 3. For added-signal trials, the abscissa reflects the correlation level prior to manipulating the visual stimulus. The $\times s$ and dotted curve represent data from an experiment conducted immediately before the "added-signal" experiment under identical psychophysical conditions except that there were no added-signal trials. $A$, An experiment in which we added $5 \%$ cor-

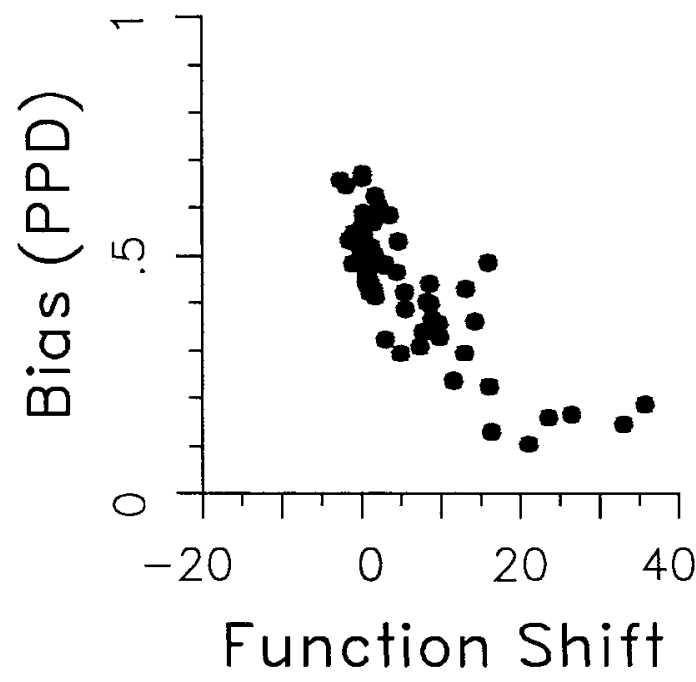

Figure 22. The relationship between choice bias on no-added-signal trials and the shift of the psychometric function. The axes are identical to those shown in Figure 18.

in which the visual stimulus was unmanipulated and the monkey was rewarded for correct choices. These data provided baseline measurcments for comparison against the monkcy's bchavior in the experiment containing added-signal trials.

Figure 21 depicts results from three psychophysical experiments in which added-signal trials contained an extra 5\% (Fig. $21 \mathrm{~A}$ ), 15\% (Fig. 21B), and 20\% (Fig. 21C) correlated dots in an arbitrarily defined preferred direction. As in the microstimulation experiments, the values on the abscissa represent stimulus direction and strength before experimental manipulation of the stimulus on added-signal trials. The $\times \mathrm{s}$ and the dotted lines represent baseline data obtained during the preliminary testing period. Open circles and dashed lines indicate the monkey's performance on no-added-signal trials, while solid circles and solid lines show performance on added-signal trials. In each experiment, the separation between the added-signal and noadded-signal psychometric functions corresponded almost exactly to the visual signal added experimentally $(5 \%, 15 \%$, and $20 \%$ correlated dots, respectively). As expected, the added-signal psychometric function was shifted leftward in comparison to the baseline data obtained during the preliminary testing period. In addition, the no-added-signal function was shifted rightward in comparison to the baseline data, indicating the induction of a null choice bias on no-added-signal trials.

It is important to realize that trials in the baseline data set ( $x$ s and dotted lines) were identical to the no-added-signal trials,

related dots to half of the trials. The distance between the no-addedsignal psychometric function and the added-signal psychometric function was $5.6 \%$ correlated dots, corresponding closely to the percentage of correlated dots added to half the trials. In the no-added-signal psychometric function, the monkey had a small null choice bias. $B$, An experiment in which we added $15 \%$ correlated dots to half of the trials. The observed shift of the psychometric function was $15.3 \%$ correlated dots. Once again, there was a null choice bias on no-added-signal trials, and there was little choice bias present prior to the experiment $(\times s$ and dotted curve). $C$, An experiment in which we added $20 \%$ correlated dots to half of the trials. The shift of the psychometric function was $21.4 \%$ correlated dots. In this experiment, a very large null choice bias is apparent on no-added-signal trials. 


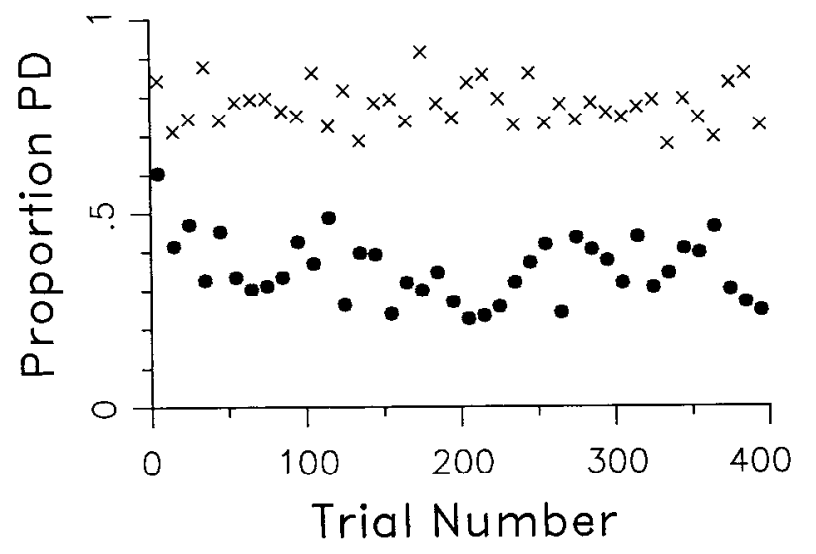

Figure 23. Time course of the monkey's choice behavior on addedsignal and no-added-signal trials in 29 psychophysical experiments. The axes are identical to those shown in Figure 10, and the criteria for including data in this analysis were identical to those for Figure 10. The $\times s$ represent added-signal trials, and the circles correspond to no-addedsignal trials.

both in the strength of the visual stimuli and in the reward contingencies. The only difference between the two was the context in which the trials were presented: the no-added-signal trials were randomly interleaved with added-signal trials, while trials from the baseline data set occurred in isolation. Thus, the null choice bias on no-added-signal trials was related to the preferred bias induced on added-signal trials. Replication of the behavioral contingencies of the microstimulation experiments therefore causes a null choice bias to develop in the absence of microstimulation.

The null choice bias observed in no-added-signal trials is similar in two further respects to the null choice bias observed in the microstimulation experiments. First, as shown in Figure 22 , the magnitude of the null choice bias in the psychophysical experiments increased proportionally to the magnitude of the shift of the psychometric function $(r=-0.811 ; p<0.0005$; compare to microstimulation data in Fig. 18). Second, the null choice bias developed very rapidly in both sets of experiments. Figure 23 shows the time course of the added-signal effect averaged across 29 psychophysical experiments. $\times$ s represent choices made on added-signal trials while the circles show performance on no-added-signal trials. The analysis was conducted in the same manner as the time course analysis of the microstimulation effect illustrated in Figure 10. On average, the null choice bias in both data sets developed fully during the first 20 50 trials of an experiment. The rapid development of the null choice bias in both microstimulation and psychophysical experiments suggests that a similar mechanism underlies the onset of the null choice bias in both cases.

The null choice bias on no-added-signal trials differed from the null choice bias in the microstimulation experiments in that it did not fully compensate for excess preferred decisions made on added-signal trials. Figure 24 shows the monkey's average choice behavior, overall, as a function of trial number in the psychophysical experiments. This analysis includes the same trials as Figure 23, but performance is pooled for added-signal and no-added-signal trials. As in the microstimulation experiments (see Fig. 20), the monkey began with a net choice bias toward the direction of the added signal. The monkey rapidly adjusted its behavior with a null choice bias on no-added-signal

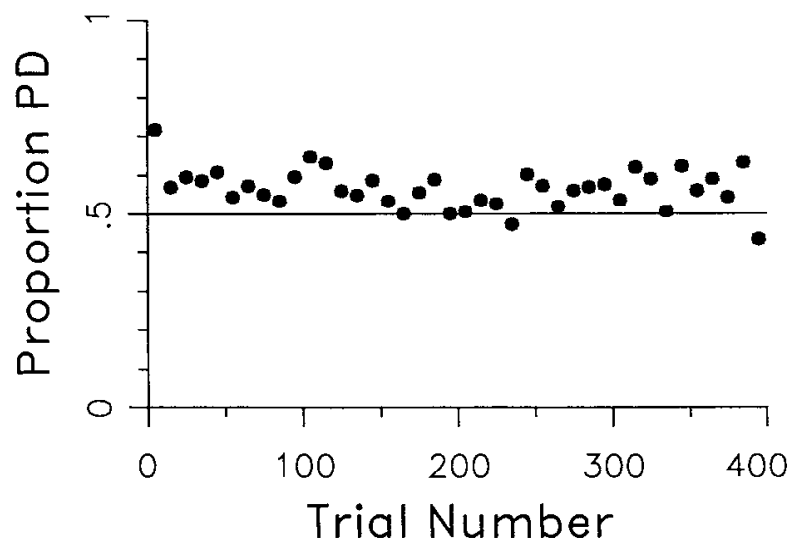

Figure 24. Time course of the monkey's overall choice behavior in the 29 psychophysical experiments. This figure pools together the addedsignal and no-added-signal trials depicted in Figure 23 and corresponds to Figure 20 in the microstimulation experiments.

trials, so that choice frequencies were more cvenly distributed between the two LEDs for the duration of the experiment. However, the steady state choice behavior hovered about $55 \%$ preferred decisions in Figure 24, instead of 50\% preferred decisions as observed in the microstimulation experiments (Fig. 20). This slight discrepancy between choice behavior and reward probabilities was largely due to experiments like that shown in Figure $21 \mathrm{C}$. In this experiment, the rightward shift of the no-addedsignal function was smaller than the leftward shift of the addedsignal function, resulting in a net preferred bias over the course of the experiment. This imprecision in probability matching was frequently observed in experiments with large amounts of added signal ( $>15 \%$ correlated dots). We have no simple explanation for the discrepancy between Figures 20 and 24 .

These psychophysical results, combined with the temporal offset microstimulation results, indicate that the null choice bias on nonstimulated trials in the microstimulation experiments resulted from a behavioral strategy such as probability matching rather than from direct physiological effects of the stimulating current. Although the behavioral paradigm employed in this study was originally selected to investigate the neural mechanisms underlying motion perception, performance on the direction discrimination task appears more complex than we anticipated. Perceptual decisions are influenced by multiple factors including the strength and direction of the visual stimulus, the presence or absence of microstimulation, and a behavioral strategy adopted by the animals in response to reward contingencies.

\section{Discussion}

Motion perception is commonly assumed to be mediated by the activity of direction selective neurons in visual cortex. The present study provides compelling evidence supporting this notion by linking the responses of direction selective neurons to perceptual judgements of motion direction. Our central finding was that electrical microstimulation applied to direction selective MT neurons can influence a monkey's performance on a direction discrimination task in a manner that is predictable from the physiological properties of the stimulated neurons. When we applied microstimulation, the direction of motion reported by monkeys was biased toward the preferred direction of neurons at the stimulation site. These effects were frequently quite 
large, dominating the animal's choice behavior even in the presence of strong motion signals of the opposite direction (e.g., Fig. $8 B$ ); these effects cannot be explained by reward incentives built into the behavioral paradigm. The results suggest that microstimulation influenced choices by inserting a directional "signal" into the cortical circuitry that underlies performance on the discrimination task.

In most experiments that yielded significant effects, the directional signal inserted by microstimulation was relatively pure, resulting in a shift of the psychometric function while preserving its shape. In a minority of experiments, however, microstimulation caused changes in the shape of the psychometric function in a manner consistent with the addition of "noise" into the neural processes encoding motion direction. In these cases, the slope of the psychometric function for stimulated trials was flatter than the slope of the nonstimulated psychometric function. Nonselective activation of multiple cortical columns that encode different directions of motion could disrupt the representation of motion in the cortex, thereby decreasing the monkeys' sensitivity to changes in the visual stimulus and flattening the psychometric function.

In the remainder of this article, we will discuss several issues relevant to the interpretation of the results. First, we consider how columnar geometry and spread of the stimulating current may affect the magnitude of microstimulation effects. Next, we discuss possible reasons for the gradual decay of the stimulation effects with time during an experiment, and we then examine various mechanisms that might underlie the microstimulation effects. Finally, we will review prior microstimulation studies of visual cortex and suggest future directions for using microstimulation to investigate the neural basis of visual perception.

Current spread. In principle, intracortical microstimulation can elicit action potentials from neurons by three mechanisms: (1) direct activation of cell bodies near the electrode tip, (2) orthodromic or antidromic activation of fibers near the electrode tip, and (3) transsynaptic activation of neurons one or more synapses away from directly activated neurons. We did not directly measure the effective extent of current spread in our experiments. Moreover, we are not aware of any previous studies concerning current spread in visual cortex. Such issues have been more extensively studied in motor systems (for reviews, see Ranck, 1975, 1981).

In primate motor cortex, a single cathodal $10 \mu \mathrm{A}$ current pulse directly activates neurons within $85 \mu \mathrm{m}$ of the electrode tip (95\% confidence intervals at $70 \mu \mathrm{m}$ and $120 \mu \mathrm{m}$ ) (Stoney et al., 1968). Trains of electrical pulses excite a larger population of neurons, predominantly by increasing transsynaptic activation (Jankowska et al., 1975; Asanuma et al., 1976), but the precise extent of transsynaptic spread is difficult to estimate. Using $10 \mu \mathrm{A}$ stimulating pulses, transsynaptic effects have been observed in physiological recordings up to $1 \mathrm{~mm}$ away from a stimulation site (Asanuma et al., 1976). In a more recent study employing optical imaging techniques in rat striate cortex, Orbach and colleagues found that $20-100 \mu \mathrm{A}$ stimulating pulses activate neurons $1 \mathrm{~mm}$ from the electrode tip (Orbach et al., 1991). Transsynaptic inhibition resulting from microstimulation has also been noted by Asanuma and Rosen (1973) and by Deyoe (E. A. Deyoe, personal communication). In addition, Hess et al. (1975) have observed that iontophoretic application of glutamate to visual cortex can generate a "halo" of inhibition up to $500 \mu \mathrm{m}$ from the site of primary excitation. Transsynaptic excitation may therefore amplify the effects of microstimulation in some circumstances, but inhibition could reduce the effects in other circumstances.

If extensive transsynaptic excitation occurs in our experiments, how can functional specificity of the microstimulation signal be preserved? Recent work by Ts'o, Gilbert, and Wiesel suggests that functionally specific columns in striate cortex have selective, excitatory connections with distant columns sharing similar physiological properties (Ts'o et al., 1986; Ts'o and Gilbert, 1988; Gilbert and Wiesel, 1989). If similar connections exist within MT, and perhaps between MT and other cortical areas, transsynaptic activation in our experiments could be restricted to neurons with physiological properties similar to those at the stimulation site. Transsynaptically elicited activity might thereby preserve the functional specificity of the microstimulation signal while increasing the number of activated neurons. In principle, an upper limit on the extent of transsynaptic spread could be provided by applying information about magnification factor and receptive field size in MT to the results of the spatial offset experiments illustrated in Figures 11-14. A reliable estimate of transsynaptic spread has not proven possible, however, because of the substantial variability in topography and magnification factor in macaque MT (Van Essen et al., 1981; Albright and Desimone, 1987; Maunsell and Van Essen, 1987).

Despite our inability to determine the extent of cortex affected by microstimulation, the precise position of the electrode tip played an important role in determining the effect of microstimulation. A particularly clear example was shown in Figure 15. Microstimulation was applied to two sites in MT, separated by only $300 \mu \mathrm{m}$. In each experiment, microstimulation biased the monkey's judgements toward the neurons' preferred direction, even though the preferred direction of neurons at each site was $180^{\circ}$ opposed. In other experiments not reported in this article, we found that changes in electrode position as small as $100 \mu \mathrm{m}$ could determine whether we obtained a large effect or no effect at all in a particular experiment. These anecdotal pieces of evidence suggest that the pattern of activity resulting from microstimulation is largely determined by the neuronal elements very close to the electrode tip. This conclusion is consistent with recent observations made by Tootell and Born (1991). They employed 2-deoxyglucose techniques to show that microstimulation of MT with parameters identical to our own causes increased metabolic activity in a restricted region of cortex near the electrode tip (half-width at half-height, $120 \mu \mathrm{m}$ ).

What determines the magnitude of the microstimulation effects? The microstimulation effects observed during this study varied widely in amplitude, ranging from no effect at all to surprisingly strong effects with equivalent visual stimuli of more than $100 \%$ correlated dots (Figs. 6, 8). The variability in magnitude of the behavioral effects was probably influenced by at least four interacting factors. First, the cortical signal generated by the stimulating current may have differed in amplitude depending upon the amount of transsynaptic excitation of nearby columns having similar physiological properties (see above). Second, the local geometry of direction columns near the electrode tip probably affected the degree of selective activation achieved by microstimulation. Direction columns in MT, like orientation columns in V1, appear to be irregular in shape (Tootell and Born, 1990), and our electrode typically crossed portions of several columns during a penetration. Since we did not know the properties of neurons outside the path of electrode travel, 
our electrode was probably near the middle of a column for some experiments but near the edge of a column for others. The imprecision in electrode placement could therefore account for much of the variability in our results since very small changes in electrode position can dramatically alter the outcome of an experiment. Third, even if the electrode was well positioned with respect to the geometry of surrounding columns, the outcome of the experiment appeared to depend somewhat on the cortical layer being stimulated. While we obtained significant effects during this study from stimulation sites in most cortical layers, six of the eight large-effect experiments marked by lesions occurred when the stimulation site was in or very near to layer 4. The result may imply that microstimulation is maximally effective when it mimics the natural inputs to a column by activating layer 4 ncurons. Finally, functional heterogeneity of columns may exist within MT in a fashion similar to the heterogeneity established in V1 and V2, where physiological properties covary with anatomical subdivisions revealed by cytochrome oxidase stains. The patchy pattern of cytochrome oxidase staining in MT (Tootell and Born, 1990, 1991) is consistent with such heterogeneity. It is conceivable, therefore, that some of our experiments failed to yield effects because the outputs of the stimulated neurons were not directed toward higher brain areas "interpreting" the translational motion of objects in the visual scene. Instead, the outputs could have been directed toward higher centers that utilize motion signals to guide pursuit eye movements or to compute optic flow patterns for judgements about the direction of self motion.

Dissipation of the microstimulation effect. Although the behavioral effect of microstimulation remained constant throughout some experiments, the effect typically waned as an experiment progressed (Fig. 10). There are several plausible physiological mechanisms that could account for the deterioration of microstimulation effects. For example, transient fatigue of, or permanent damage to, neurons near the stimulation site could account for the decline in efficacy. Small shifts in electrode position in the brain could also account for some portion of the decline. During single neuron recording experiments of similar duration (about $1 \mathrm{hr}$ ), we know that the position of an electrode with respect to the brain can gradually change since it is frequently necessary to withdraw the electrode by as much as $200 \mu \mathrm{m}$ to maintain isolation of the cell's action potential. As stated above, changes in electrode position of this magnitude can dramatically influence the outcome of a stimulation experiment. We are unable to monitor such shifts during a microstimulation experiment since the electrode is connected to a stimulator rather than to a recording amplifier. Recordings conducted after a microstimulation experiment generally yielded weaker visual responses in comparison to preexperiment levels. This observation is consistent with neuronal fatigue, damage, or shifts in electrode position, although an apparent decrease in responsiveness could have resulted from electrode etching, which we consistently observed after microstimulation experiments.

Although physiological factors probably caused the dissipation in microstimulation effects, it is conceivable that a monkey could adopt a behavioral strategy that gradually reduces the effect of microstimulation during an experiment. The motivation for implementing this strategy would be to improve reward rate since large microstimulation effects were associated with a lower percentage of correct judgements (Fig. 5). 'The improve- ment in reward rate would be brought about by decreasing the proportion of preferred decisions made on stimulated trials while either increasing or leaving relatively unchanged the proportion of preferred choices on nonstimulated trials. To accomplish this, a monkey would have to be able to distinguish between stimulated and nonstimulated trials and adjust its choices accordingly. Our experiments suggest, however, that the monkeys do not distinguish between stimulated and nonstimulated trials. If the animals could make such a distinction and alter their choice strategy to their advantage, we would expect these changes to be cumulative with time. Thus, we would expect fewer and weaker microstimulation effects as the monkeys learned the new strategy and employed it more regularly and effectively. However, we have now conducted more than 100 microstimulation experiments over the course of 6-18 months in each of two monkeys (monkey $\mathrm{E}$ and monkey $\mathrm{J}$ ), and we have observed no decrease in the frequency or intensity of microstimulation effects. In fact, our success rate has increased modestly because we have become more adept at positioning the stimulating electrode. We therefore infer that the monkeys do not alter their choice strategy based on a subjective distinction between stimulated and nonstimulated trials. Dissipation in the microstimulation effects is probably mediated by physiological causes.

Probability matching and the null choice bias. Data presented in section III of Results indicate that the null choice bias on nonstimulated trials can be accounted for by a behavioral strategy in which the monkeys match their perceptual choices to the expected rewarded frequencies of the two possible choices (Fig. 20). This type of probability matching has been studied extensively in humans by experimental psychologists (for reviews, scc Estes, 1964; Atkinson et al., 1965). These studies have shown that in the absence of a stimulus that cues the correct choice, human subjects will match their choices to the expected reward frequencies. Probability matching tends to be particularly precise when the subject believes that the correct choice is actually cued by the stimulus, even though the information contained in the stimulus is not useful (Goodnow, 1955; Goodnow and Postman, 1955). This example is very similar to the situation facing our monkeys when making choices concerning motion direction at correlation levels below psychophysical threshold such as $0 \%$ correlation. There is very little information in the visual stimulus about the correct choice, yet the monkeys still try to discriminate correctly the direction of motion in the stimulus. It is not surprising, therefore, that under such conditions our monkeys base their decisions in part upon the reward contingencies of the experiment and that they match perceptual choices to the corresponding choice probabilities.

Comparison to prior microstimulation studies. Electrical stimulation of the brain has been a primary tool in the investigation of motor systems for over a century. In sensory systems, however, electrical stimulation has been employed relatively rarely. In a series of investigations begun in the 1920 s, surface electrodes were used to stimulate the visual cortex of blind volunteers or of seeing patients undergoing surgery for epilepsy (e.g., Penfield and Rasmussen, 1952; Penfield and Jasper, 1954; Brindley and Lewin, 1968; Brindley, 1973, 1982; Dobelle et al., 1979; Evans et al., 1979; Girvin et al., 1979). Subjects reported seeing a point of light, or phosphene, in a region of space that was topographically consistent with the known map of visual space in human striate cortex. These pioneering studies suggested that electrical stimulation could be profitably used to 
investigate visual function even though their methods were too crude to activate functionally specific circuits within the cortex. More recently, Deyoe reported that monkeys could be trained to detect microstimulation pulses as weak as $1 \mu \mathrm{A}$ applied intracortically in striate cortex (Deyoe, 1983). These monkeys presumably detected the presence of microstimulation in striate cortex by "seeing" phosphenes of the sort reported by human patients. The study of Deyoe is consistent with our results in that weak stimulating currents can achieve behavioral significance to an animal.

To our knowledge, the first attempt to use microstimulation to study the function of directionally specific circuits in MT was a recent study by Komatsu and Wurtz (1989). Prior lesion studies suggested that motion information encoded by neurons in MT and MST contributes to the guidance of smooth-pursuit eye movements (Newsome et al., 1985; Dursteler et al., 1987). Komatsu and Wurtz therefore attempted to influence pursuit eye movements by stimulating these areas. They obtained directionally specific effects on the maintenance of pursuit with stimulation of MST and foveal MT, but stimulation of extrafoveal MT yielded only nonspecific effects on pursuit initiation reminiscent of those caused by MT lesions. We have recently found that large stimulating currents $(80 \mu \mathrm{A})$ similar to those used by Komatsu and Wurtz impair performance on our direction discrimination task, and we believe that this impairment results from nonspecific activation of multiple direction columns (Murasugi et al., 1991). A promising experiment would test whether small stimulating currents on the order of $10 \mu \mathrm{A}$ could influence pursuit eye movements in a directionally specific manner.

Mechanisms underlying the effect. A simple description of the neural processes underlying performance on our task could include three stages. First, the brain encodes the relevant sensory information about the visual stimulus. Second, the sensory signals are "evaluated" and a decision made as to the direction of stimulus motion. Finally, a saccadic eye movement is programmed and executed to indicate a choice. This description of the task suggests that microstimulation could in principle intervene at any of these three stages: (1) sensory processing, (2) decisional mechanisms, or (3) motor circuits. We currently favor the first hypothesis, since there is considerable evidence implicating MT in processing sensory signals related to motion information. We cannot, however, rule out the possibility that microstimulation directly affects a decisional mechanism. In contrast, there is substantial evidence that microstimulation in MT does not exert its primary effect on motor circuits. We will consider each of these possibilities in turn.

If microstimulation influences the processing of sensory signals, it is natural to wonder what an animal sees during microstimulation. Although we obviously do not know the answer to this question, the data suggest certain conclusions. The fact that the monkeys do not seem to develop different choice strategies for stimulated and nonstimulated trials suggests that microstimulation does not generate moving phosphenes that are readily distinguishable from the random dot stimuli. It also seems unlikely that microstimulation adds features to the scene that appear subjectively as moving random dots. Perception of randomly distributed points of light like our random dot patterns must certainly involve complex patterns of activity across a large expanse of cortex, probably including more primary visual areas such as V1 and V2. Instead, a plausible hypothesis is that microstimulation evokes a subjective sensation of motion like that experienced during the motion aftereffect, or waterfall illusion. Motion aftereffects can be very powerful, generating a robust motion percept even if the observer is aware cognitively that the perceived object cannot possibly be moving. Motion therefore appears to be a quality that can be computed independently within the brain and "assigned" to patterned objects in the environment. This sort of assignment of motion quality to the random dot patterns would be consistent with our observations. There would be no obvious visual cue that the animal could use to distinguish stimulated from nonstimulated trials, but the subjective sensations of motion could account for the influence of microstimulation on the animal's choice behavior in the direction discrimination task. Incisive tests of such hypotheses must, however, involve experiments with human subjects who can describe what they see. Interestingly, it has recently been reported that crude motion percepts can be elicited with electrical stimulation of human parietooccipital cortex (G. T. Plant, personal communication).

At present, we cannot exclude the possibility that microstimulation as applied in our paradigm influences decisional mechanisms without affecting what the animal actually sees. Decisions concerning the identity and motion of objects in the environment are important integrative mechanisms linking sensory processing to action, but we presently have little insight into the neurobiological substrate of these decisional processes. The results of the spatial offset experiments (Figs. 11-14) suggest, however, that microstimulation does not affect a generalized decisional mechanism. If microstimulation directly affects a decisional mechanism, therefore, the decisional mechanism must receive inputs preferentially from neurons encoding motion in a restricted region of the visual field. A satisfactory account of the animal's behavior would require several such mechanisms operating in parallel at different regions of the visual field. Physiological recording from brain areas "downstream" from MT may eventually yield insight concerning the nature of decisional mechanisms operating during performance on our paradigm.

A considerable body of evidence suggests that microstimulation in MT does not exert direct effects on the motor circuits that produce saccadic eye movements. Physiological recordings during pursuit and saccadic eye movements have failed to yield evidence of motor signals in MT (Newsome et al., 1988), and lesions of MT have no effect on saccadic eye movements to stationary targets (Newsome et al., 1985). Komatsu and Wurtz (1989) found that large stimulating currents $(100 \mu \mathrm{A})$ applied to foveal MT can increase the latency of saccades to stationary targets, but this effect was not observed in extrafoveal MT where our experiments were conducted.

In addition, several observations made during the present study support the conclusion that microstimulation of MT did not produce eye movements by acting directly on motor circuits. First, we did not observe any short-latency saccades associated with microstimulation of $\mathrm{MT}$, whether microstimulation was applied during the intertrial interval or during the fixation interval. In fact, execution of the saccade in our task occurred more than $1 \mathrm{sec}$ after the onset of the train of electrical pulses. Latencies of this length would not be expected from direct activation of motor circuits. Second, for the original 62 experiments (see Fig. $6 A$ ), we compared the frequency of inappropriate breaks in fixation on stimulated and nonstimulated trials. There was no significant difference between the two $\left(\chi^{2}\right.$ test, $\left.p>0.2\right)$, implying that microstimulation did not cause short-latency eye 
movements during the fixation interval. Third, the microstimulation effects were larger in amplitude on trials containing weak motion signals (low correlations) than on those containing strong motion signals (high correlations). This stimulus dependence suggests that the microstimulation effects are specifically related to the changing visual inputs received during the task rather than to motor outputs produced, which remain constant during the task. Finally, we obtained data contradicting two specific hypotheses concerning possible motor effects of microstimulation in MT: microstimulation does not simply increase the probability of making an eye movement toward the receptive field location of the stimulated neurons (Fig. 15), nor does microstimulation simply increase the likelihood of an eye movement in the same direction as that preferred by neurons at the stimulation site (Fig. 16). We therefore find no reasonable grounds for postulating that the microstimulation effects observed during this study are due to direct effects on motor circuits.

$M T$ and the motion pathway. Zeki and colleagues first discovered that most neurons in a restricted region of the STS yielded directionally selective responses to visual stimuli, and they proposed that this area of visual cortex was specialized for processing motion information (Dubner and Zeki, 1971; Zeki, $1974,1975,1978$ ). Building on Zeki's work, considerable evidence indicates that this area (MT, or V5) is but one locus on a pathway that selectively analyzes visual motion information (Ungerleider and Desimone, 1986; Maunsell and Newsome, 1987). The information extracted and encoded within this pathway is utilized for a number of purposes related to motion vision. For example, lesion and single-unit recording studies implicate MT and a higher area of the motion pathway, MST, in the guidance of pursuit eye movements (Newsome et al., 1985, 1988; Dursteler et al., 1987; Dursteler and Wurtz, 1988), discrimination of motion direction (Newsome and Pare, 1988; Newsome et al., 1989a,b), detection of shearing motion and three-dimensional structure-from-motion (Siegel and Andersen, 1986), and speed discrimination (Merigan et al., 1991; Vandenbussche et al., 1991). Additional single-unit recordings have shown that these areas synthesize lower-order motion signals to represent the motion of complex, two-dimensional patterns (Movshon et al., 1985; Rodman and Albright, 1989), analyze motion in optic flow patterns (Tanaka et al., 1986, 1989; Tanaka and Saito, 1989; Graziano et al., 1990; Duffy and Wurtz, 1991a,b; Lagae et al., 1991), and potentially distinguish figure from ground on the basis of motion cues (Allman et al., 1985). Some neurons in MT may also reflect the modulation of motion signals during binocular rivalry (Logothetis and Schall, 1989).

The results of this study confirm and extend prior work by directly linking the activity of direction selective neurons to perceptual judgments of motion direction. Although we applied microstimulation directly to MT in all of our experiments, the resulting pattern of neural activity may have been quite extensive because of the rich network of connections between MT and other areas within the motion pathway, such as V1, V2, V3 and MST. Whether this circuit of neurons can be activated by microstimulation of other areas within the motion pathway is an important question for future experiments. Areas V3 and MST seem likely candidates for such studies, since those areas contain substantial populations of direction-selective neurons (Van Essen et al., 1981; Ungerleider and Desimone, 1986; Felleman and Van Essen, 1987).

Assumptions linking physiological properties to perceptual experience form the basis for many lines of investigation in visual neuroscience. The present study suggests that these linking hypotheses can actually be tested experimentally and confirmed. A natural extension of this work is to apply the same basic approach to the study of circuits that mediate aspects of visual perception other than motion. In principle, the microstimulation technique is applicable to the analysis of function in any circuit in which neurons with similar physiological properties are segregated into columns or large clusters. Any investigation would presuppose, however, that hypotheses concerning function are sufficiently well developed from single-unit recordings that appropriate psychophysical tests can be designed. Given present physiological knowledge, appropriate candidates for future investigation are circuits that encode orientation, color, and disparity.

\section{References}

Albright TD (1984) Direction and orientation selectivity of neurons in visual area MT of the macaque. J Neurophysiol 52:1106-1130.

Albright TD, Desimone R (1987) Local precision of visuotopic organization in the middle temporal area (MT) of the macaque. Exp Brain Res 65:582-592.

Albright TD, Desimone R, Gross CG (1984) Columnar organization of directionally selective cells in visual area MT of macaques. J Neurophysiol 51:16-31.

Allman JM, Meizin F, McGuinness E (1985) Direction and velocityspecific responses from beyond the classical receptive field in the middle temporal visual area (MT). Perception 14:105-126.

Anstis SM (1980) The perception of apparent movement. Philos Trans R Soc Lond [Biol] 290:153-168.

Asanuma H, Rosen I (1973) Spread of mono- and polysynaptic connections within cat's motor cortex. Exp Brain Res 16:507-520.

Asanuma H, Arnold A, Zarzecki P (1976) Further study on the excitation of pyramidal tract cells by intracortical microstimulation. Exp Brain Res 26:443-461.

Atkinson RC, Bower GH, Crothers EJ (1965) An introduction to mathematical learning theory. New York: Wiley.

Baker JF, Petersen SE, Newsome WT, Allman JM (1981) Visual response properties of neurons in four extrastriate visual areas of the owl monkey (Aotus trivirgatus). J Neurophysiol 45:397-416.

Barlow HB, Blakemore C, Pettigrew JD (1967) The neural basis of binocular depth discrimination. J Physiol (Lond) 193:327-342.

Braddick OJ (1974) A short-range process in apparent movement. Vision Res 14:519-527.

Brindley GS (1973) Sensory effects of electrical stimulation of the visual and paravisual cortex in man. In: Handbook of Sensory Physiology (Jung R, ed), Vol VII, Chap 3B, pp 583-594. Berlin: Springer.

Brindlcy GS (1982) Effects of electrical stimulation of the visual cortex. Hum Neurobiol 1:281-283.

Brindley GS, Lewin WS (1968) The sensations produced by electrical stimulation of the visual cortex. J Physiol (Lond) 196:479-493.

Cox DR (1970) Analysis of binary data. London: Methuen.

Crist CF, Yamasaki DSG, Komatsu H, Wurtz RH (1988) A grid system and microsyringe for single cell recording. J Neurosci Methods 26:117-122.

Damasio AR, Benton AL (1979) Impairments of hand movements under visual guidance. Neurology 29:170-178.

Damasio AR, Damasio H, Van Hoesen GW (1982) Prospagnosia: anatomic basis and behavioral mechanisms. Neurology 32:331-341.

De Valois RL, Yund EW, Hepler N (1982) The orientation and direction selectivity of cells in the macaque visual cortex. Vision Res 22:531-544.

Deyoe EA (1983) An investigation in the awake macaque of the threshold for detection of electrical currents applied to striate cortex: psychophysical properties and laminar differences. PhD thesis, University of Rochester.

Dobelle WH, Turkel J, Henderson DC, Evans JR (1979) Mapping the representation of the visual field by electrical stimulation of human visual cortex. Am J Ophthalmol 88:727-735.

Dubner R, Zeki SM (1971) Response properties and receptive fields of cells in an anatomically defined region of the superior temporal sulcus. Brain Res 35:528-532. 
Duffy CJ, Wurtz RH (1991a) Sensitivity of MST neurons to optic flow stimuli. I. A continuum of response selectivity of large-field stimuli. J Neurophysiol 65:1329-1345.

Duffy CJ, Wurtz RH (1991b) Sensitivity of MSI neurons to optic flow stimuli. II. Mechanisms of response revealed by small-field stimuli. J Neurophysiol 65:1346-1359.

Dursteler MR, Wurtz RH (1988) Pursuit and optokinetic deficits following chemical lesions of cortical areas MT and MST. J Neurophysiol 60:940-965.

Dursteler MR, Wurtz RH, Newsome WT (1987) Directional pursuit deficits following lesions of the foveal representation within the superior temporal sulcus of the macaque monkey. $J$ Neurophysiol 57: $1262-1287$.

Estes WK (1964) Probability learning. In: Categories of human learning (Melton AW, ed), pp 89-128. New York: Academic.

Evans JR, Gordon J, Abramov I, Mladejovsky MG, Dobelle WH (1979) Brightness of phosphenes elicited by electrical stimulation of human visual cortex. Sens Processes 3:82-94.

Evarts EV (1966) Methods for recording activity of individual neurons in moving animals. Methods Med Res 11:241-250.

Evarts EV (1968) A technique for recording activity of subcortical neurons in moving animals. Electroencephalogr Clin Neurophysiol 24:83-86.

Felleman DJ, Van Essen DC (1987) Receptive field properties of neurons in area V 3 of macaque monkey extrastriate cortex. J Neurophysiol 57:889-920.

Fischer B, Poggio GF (1979) Depth sensitivity of binocular cortical neurons of behaving monkeys. Proc R Soc Lond [Biol] 204:409-414.

Fuchs AF, Robinson DA (1966) A method for measuring horizontal and vertical eye movement chronically in the monkey. J Appl Physiol 21:1068-1070.

Gallyas F (1979) Silver staining of myelin by means of physical development. Neurol Res 1:203-209.

Gilbert CD, Wiesel TN (1989) Columnar specificity of intrinsic horizontal and cortico-cortical connections in cat visual cortex. J Neurosci 9:2432-2442.

Girvin JP, Evans JR, Dobelle WH, Mladejovsky MG, Henderson DC, Abramov I, Gordon J, Turkel J (1979) Electrical stimulation of human visual cortex: the effect of stimulus parameters on phosphene threshold. Scns Processes 3:66-81.

Goodnow JJ (1955) Determinants of choice-distribution in two-choice situations. Am J Psychol 68:106-116.

Goodnow JJ, Postman L (1955) Probability learning in a problemsolving situation. J Exp Psychol 49:16-22.

Graziano M, Andersen R, Snowden R (1990) Stimulus selectivity of neurons in macaque MST. Soc Neurosci Abstr 16:6.

Hammond P, Mouat GSV, Smith AT (1985) Motion after-effects in cat striate cortex elicited by moving gratings. Exp Brain Res 60:411416.

Hammond P, Mouat GSV, Smith AT (1986) Motion after-effects in cat striate cortex elicited by moving texture. Vision Res 26:10551060 .

Hays AV, Richmond BJ, Optican LM (1982) A UNIX-based multiple process system for rcal-time data acquisition and control. WESCON Conf Proc 2:1-10.

Hess R, Negishi K, Creutzfeldt O (1975) The horizontal spread of intracortical inhibition in the visual cortex. Exp Brain Res 22:415419.

Hubel DH, Wiesel TN (1962) Receptive fields, binocular interaction and functional architecture in the cat visual system. J Physiol (Lond) 160:106-154.

Hubel DH, Wiesel TN (1968) Receptive fields and functional architecture of monkey striate cortex. J Physiol (Lond) 195:215-243.

Jankowska E, Padel Y, Tanaka R (1975) The mode of activation of pyramidal tract cells by intracortical stimuli. J Physiol (Lond) 249: $617-636$.

Joynt RJ, Honch GW, Rubin AJ, Trudell RG (1985) Occipital lobe syndromes. Handb Clinical Neurol 1:45-62.

Judge SJ, Richmond BJ, Chu FC (1980). Implantation of magnetic search coils for measurement of eye position: an improved method. Vision Res 20:535-538.

Komatsu H, Wurtz RH (1989) Modulation of pursuit eye movements by stimulation of cortical areas MT and MST. J Neurophysiol 62: $31-47$.
Lagae L, Dengke X, Raiguel S, Maes H, Orban G (1991) Position invariance of optic flow component selectivity differentiates monkey MST and FST cells from MT cells. Assoc Res Vis Ophthalmol Abstr 32:823.

Lennie P, Krauskopf J, Sclar G (1990) Chromatic mechanisms in striate cortex of macaque. J Neurosci 10:649-669.

Livingstone MS, Hubel DH (1984) Anatomy and physiology of a color system in the primate visual cortex. J Neurosci 4:309-356.

I ogothetis NK, Schall JD (1989) Neuronal correlates of subjective visual perception. Science 245:761.

Maunsell JHR, Newsome WT (1987) Visual processing in monkey extrastriate cortex. Annu Rev Neurosci 10:363-401.

Maunsell JHR, Van Essen DC (1983) Functional properties of neurons in middle temporal visual area of the macaque monkey. I. Selectivity for stimulus direction, speed and orientation. J Neurophysiol 49: $1127-1147$

Maunsell JHR, Van Essen DC (1987) Topographic organization of the middle temporal visual area in the macaque monkey: representational biases and the relationship to callosal connections and myeloarchitectonic boundaries. J Comp Neurol 266:535-555.

Meadows JC (1974a) The anatomical basis of prosopagnosia. J Neurol Neurosurg Psychiatr 37:489-501.

Meadows JC (1974b) Disturbed perception of colours associated with localized cerebral lesions. Brain 97:615-632.

Merigan WH, Pasternak T, Polashenski W, Maunsell JHR (1991) Permanent deficits in speed discrimination after MT/MST lesions in a macaque monkey. Assoc Res Vis Ophthalmol Abstr 32:824.

Mikami A, Newsome WT, Wurtz RH (1986) Motion selectivity in macaque visual cortex: II. Spatio-temporal range of directional interactions in MT and V1. J Neurophysiol 55:1328-1339.

Mishkin M, Ungerleider LG, Macko KA (1983) Object vision and spatial vision: two cortical pathways. Trends Neurosci 6:414-417.

Morgan MJ, Ward R (1980) Conditions for motion flow in dynamic visual noise. Vision Res 20:431-435.

Movshon JA, Adelson EH, Gizzi MS, Newsome WT (1985) The analysis of moving visual patterns. In: Pattern recognition mechanisms (Chagas C, Gattass R, Gross C, eds), pp 117-151. New York: Springer.

Murasugi CM, Salzman CD, Newsome WT (1991) Microstimulation of visual area MT during motion discriminations: II. Effects of varying pulse amplitude and frequency. Assoc Res Vis Ophthalmol Abstr 32: 822.

Nakayama K (1981) Differential motion hyperacuity under conditions of common image motion. Vision Res 21:1475-1482.

Newsome WT, Pare EB (1986) MT lesions impair discrimination of direction in a stochastic motion display. Soc Neurosci Abstr 12:1183.

Newsome WT, Pare EB (1988) A selective impairment of motion perception following lesions of the middle temporal visual area (MT). J Neurosci 8:2201-2211.

Newsome WT, Wurtz RH, Dursteler MR, Mikami A (1985) Deficits in visual motion processing following ibotenic acid lesions of the middle temporal visual area of the macaque monkey. J Neurosci 5: $825-840$.

Newsome WT, Wurtz RH, Komatsu H (1988) Relation of cortical areas MT and MST to pursuit eye movements. II. Differentiation of retinal from extraretinal inputs. J Neurophysiol 60:604-620.

Newsome WT, Britten KH, Movshon JA (1989a) Neuronal correlates of a perceptual decision. Nature 341:52-54.

Newsome WT, Britten KH, Movshon JA, Shadlen M (1989b) Single neurons and the perception of visual motion. In: Neural mechanisms of visual perception. Proceedings of the retina research foundation (Lam DM-K, Gilbert CD, eds), pp 171-198. The Woodlands, TX: Portfolio.

Newsome WT, Britten KH, Salzman CD, Movshon JA (1990) Neuronal mechanisms of motion perception. Cold Spring Harbor Symp Quant Biol 55:697-705.

Newsome WT, Salzman CD, Murasugi CM, Britten KH (1991) Manipulating perceptual decisions by microstimulation of extrastriate visual cortex. In: Representations of vision: trends and tacit assumptions in vision research (Gorea A, ed), pp 125-139. Cambridge: Cambridge UP.

Orbach HS, Felleman DJ, Ribak EN, Van Essen DC (1991) Visualization of cortical connections with voltage sensitive dyes. In: Analysis and modeling of neural systems (Eeckman F, ed), in press. Norwell, MA: Kluwer. 
Pantlc A (1974) Motion aftereffect magnitude as a measure of the spatiotemporal response properties of direction-sensitive analyzers. Vision Res 14:1229-1236.

Pearlman AL, Birch J, Meadows JC (1979) Cerebral colour blindness: an acquired defect in hue discrimination. Ann Neurol 5:253-261.

Penfield W, Jasper H (1954) Epilepsy and the functional anatomy of the human brain. London: Churchill.

Penfield W, Rasmussen T (1952) The cerebral cortex of man. New York: Macmillan.

Petersen SE, Baker JF, Allman JM (1985) Direction-specific adaptation in area MT of the owl monkey. Brain Res 346:146-150.

Plant GT (1990) Impaired speed discrimination restricted to the hemifield contralateral to an occipitoparietal surgical resection. Soc Neurosci Abstr 16:5.

Ranck JB Jr (1975) Which elements are excited in electrical stimulation of mammalian central nervous system: a review. Brain Res 98: 417-440.

Ranck JB Jr (1981) Extracellular stimulation. In: Electrical stimulation research techniques (Patterson MM, Kesner RP, eds), pp 1-36. San Diego: Academic.

Ratcliff G, Davies-Jones AB (1972) Defective visual localization in focal brain wounds. Brain 95:49-60.

Robinson DA (1963) A method of measuring eye movement using a scleral search coil in a magnetic field. IEEE Trans Biomed Eng 10: 137-145.

Rodman HR, Albright TD (1989) Single-unit analysis of pattern-motion selection properties in the middle temporal visual area (MT). Exp Brain Res 75:53-64.

Salzman CD, Newsome WT (1991) Microstimulation of visual area MT during motion discriminations: I. Effects on choice bias. Assoc Res Vis Ophthalmol Abstr 32:822.

Salzman CD, Britten KH, Newsome WT (1990a) Cortical microstimulation influences perceptual judgements of motion direction. Nature 346:174-177.

Salzman CD, Murasugi CM, Newsome WT (1990b) Perceptual effects of cortical microstimulation are spatially and temporally restricted. Soc Neurosci Abstr 16:5.

Schiller PH, Finlay SF, Volman J (1976) Quantitative studies of singlecell properties in monkey striate cortex. I. Spatio-temporal organization of receptive fields. J Neurophysiol 39:1288-1319.

Siegel RM, Andersen RM (1986) Motion perceptual deficits following ibotenic acid lesions of the middle temporal area (MT) in the behaving rhesus monkey. Soc Neurosci Abstr 12:1183.

Stoney SD Jr, Thompson WD, Asanuma H (1968) Excitation of pyramidal tract cells by intracortical microstimulation: effective extent of stimulating current. J Neurophysiol 31:659-669.

Tanaka K, Saito H (1989) Analysis of motion of the visual field by directions, expansion/contraction and rotation cells clustered in the dorsal part of the medial superior temporal area of the macaque monkey. J Neurophysiol 62:626-641.

Tanaka K, Hikosaka H, Saito H, Yukie Y, Fukada Y, Iwai E (1986) Analysis of local and wide field movements in the superior temporal visual areas of the macaque monkey. J Neurosci 6:134-144.

Tanaka K, Fukada Y, Saito H (1989) Underlying mechanisms of the response specificity of expansion/contraction and rotation cells in the dorsal part of the medial superior temporal area of the macaque monkey. J Neurophysiol 62:642-656.

Teller DY (1984) Linking propositions. Vision Res 24:1233-1246.

Tootell RBH, Born RT (1990) Patches and direction columns in primate area MT. Assoc Res Vis Ophthalmol Abstr 31:238.

Tootell RBH, Born RT (1991) Architecture of primate area MT. Soc Neurosci Abstr 17:524.

Ts'o DY, Gilbert CD (1988) The organization of chromatic and spatial interactions in the primate striate cortex. J Neurosci 8:1712-1727.

Ts'o DY, Gilbert CD, Wiesel TN (1986) Relationships between horizontal interactions and functional architecture in cat striate cortex as revealed by cross-correlation analysis. J Neurosci 6:1160-1170.

Ungerleider LG, Desimone R (1986) Cortical connections of visual area MT in the macaque. J Comp Neurol 248:190-222.

Ungerleider LG, Mishkin M (1982) Two cortical visual systems. In: Analysis of visual behavior (Ingle DJ, Goodale MA, Mansfield RJ, eds), pp 549-580. Cambridge, MA: MIT Press.

Vandenbussche E, Saunders RC, Orban GA (1991) MT lesions impair monkey speed discrimination. Assoc Res Vis Ophthalmol Abstr 32: 823.

Van Essen DC, Maunsell JHR, Bixby JL (1981) The middle temporal visual arca in the macaque: mycloarchitccturc, connections, functional properties and topographic representation. J Comp Neurol 199: 293-326.

Vautin RG, Berkley MA (1977) Responses of single cells in cat visual cortex to prolonged stimulus movement. J Neurophysiol 40:10511065.

Williams DW, Sekuler R (1984) Coherent global motion percepts from stochastic local motions. Vision Res 24:55-62.

Zeki SM (1974) Functional organization of a visual area in the posterior bank of the superior temporal sulcus of the rhesus monkey. J Physiol (Lond) 236:549-573.

ZekiSM (1975) The functional organization of projections from striate to prestriate visual cortex in the rhesus monkey. Cold Spring Harbor Symp Quant Biol 40:591-600.

Zcki SM (1978) Uniformity and diversity of structure and function in rhesus monkey prestriate visual cortex. J Physiol (Lond) 277:273290.

Zihl J, Von Cramon D, Mai N (1983) Selective disturbance of movement vision after bilateral brain damage. Brain 106:313-340. 